\title{
Defect activity in metal halide perovskites with wide and narrow bandgap
}

\author{
Yang Zhou ${ }^{1,5}$, Isabella Poli ${ }^{1,5}$, Daniele Meggiolaro ${ }^{2}$, Filippo De Angelis $\mathbb{1}^{2,3,4}$ and \\ Annamaria Petrozza ${ }^{1 凶}$
}

Abstract | Metal halide perovskites (MHPs) constitute a rich library of materials with huge potential for disruptive optoelectronic technologies. Their main strength comes from the possibility of easily tuning their bandgap to integrate them in devices with different functionalities - in principle. In reality, this cannot be achieved yet. In fact, whereas defect tolerance can be claimed for MHPs with a bandgap of about $1.6 \mathrm{eV}$, the model system that is the object of intense investigations, MHPs with lower and higher bandgaps are far from being defect-tolerant. These materials show various forms of instabilities that are mainly driven by strong defect activity. Here we critically assess the most recent advances in elucidating the physical and chemical activity of defects in both high-bandgap and low-bandgap MHPs, while correlating it to performance and stability losses, especially for solar cells, the driving application for these materials. We also provide an overview of the strategies so far implemented to eventually overcome the remaining materials-based and device-based challenges.

${ }^{1}$ Center for Nano Science and Technology@PoliMi, Istituto Italiano di Tecnologia, Milan, Italy.

${ }^{2}$ Computational Laboratory for Hybrid/Organic Photovoltaics (CLHYO), Istituto CNR di Scienze e Tecnologie Chimiche "Giulio Natta" (CNR- SCITEC), Perugia, Italy.

${ }^{3}$ Department of Chemistry, Biology and Biotechnology, University of Perugia, Perugia, Italy.

${ }^{4}$ CompuNet, Istituto Italiano di Tecnologia, Genova, Italy.

${ }^{5}$ These authors contributed equally: Yang Zhou,

Isabella Poli.

凶e-mail:

annamaria.petrozza@iit.it

https://doi.org/10.1038/

s41578-021-00331-x
Solar cells based on metal halide perovskites (MHPs) have shown an unprecedented rise in efficiency in the past 10 years or so. The power conversion efficiency (PCE) of these solar cells has rocketed from a bare initial $3.8 \%{ }^{1}$ to a currently certified $25.5 \%$, outcompeting established thin-film technologies such as polycrystalline silicon, cadmium telluride and copper indium gallium (di) selenide solar cells. MHPs have the generic chemical formula $\mathrm{ABX}_{3}$, with the mono-positive $\mathrm{A}^{+}$cations - mainly methylammonium $\left(\mathrm{MA}^{+}\right)$, formamidinium $\left(\mathrm{FA}^{+}\right), \mathrm{Cs}^{+}$ or combinations thereof - filling the cavities between the $\mathrm{BX}_{6}^{4-}$ octahedra $\left(\mathrm{B}=\mathrm{Sn}^{2+}\right.$ or $\mathrm{Pb}^{2+} ; \mathrm{X}=\mathrm{I}^{-}, \mathrm{Br}^{-}$or $\left.\mathrm{Cl}^{-}\right)$, which constitute a $3 \mathrm{D}$ network through corner-sharing. The versatility of the chemical composition of MHPs enables the fabrication of semiconductors with different bandgaps that span from about $1.2 \mathrm{eV}$ to the entire visible region, up to $3 \mathrm{eV}$. The most efficient solar energy harvesting has so far been achieved by MHPs with a bandgap of 1.5-1.6 eV. However, according to the Shockley-Queisser (SQ) analysis ${ }^{2}$, the optimum bandgap to achieve the maximum theoretical efficiency of over 30\% with a single-junction solar cell falls between $1.1 \mathrm{eV}$ and $1.4 \mathrm{eV}$. The absorption of MHPs can be extended to the near-infrared region by partly substituting $\mathrm{Pb}$ with $\mathrm{Sn}$ on the B-site to obtain narrow-bandgap (NB) materials, whereas halide substitution of I with $\mathrm{Br}$ and of $\mathrm{Br}$ with $\mathrm{Cl}$ on the $\mathrm{X}$-site leads to wide-bandgap
(WB) materials. Such bandgap tunability is highly advantageous for the development of a new materials platform for optoelectronic and photonic applications. This is strikingly evident in the photovoltaic field, where MHPs may allow researchers to design solar cells based on very similar device architecture with optimum SQ bandgap, to target indoor photovoltaics, to fabricate tandem devices or to drive electrochemical reactions. Despite the impressive progress on both NB and WB MHPs, there are still many open questions about the role of defects on the stability and performance of tunable perovskite absorbers. On the one hand, WB MHP solar cells suffer from substantial open-circuit voltage $\left(V_{\mathrm{oc}}\right)$ loss that limits their photovoltaic performance ${ }^{3-19}$ (FIG. 1a) and that is suggested to originate from halide segregation ${ }^{20}$. On the other hand, the highest PCE achieved with NB MHP solar cells is $21.7 \%^{12}$, which is substantially lower than that of mid-range-bandgap MHPs, in spite of a bandgap closer to the optimum SQ value. The main limitation of Sn-containing MHPs is the strong $\mathrm{p}$-doping, which increases carrier recombination, and the high tendency of $\mathrm{Sn}^{2+}$ to oxidize to $\mathrm{Sn} 4^{+21}$. This process is in part due to external agents, such as air or humidity, and it may be intrinsically driven by defects that create the environment for material degradation, causing severe short-circuit current $\left(J_{\mathrm{sc}}\right)$ loss that limits the photovoltaic performance $\mathrm{e}^{11-18,22}$ (FIG. 1 b). 

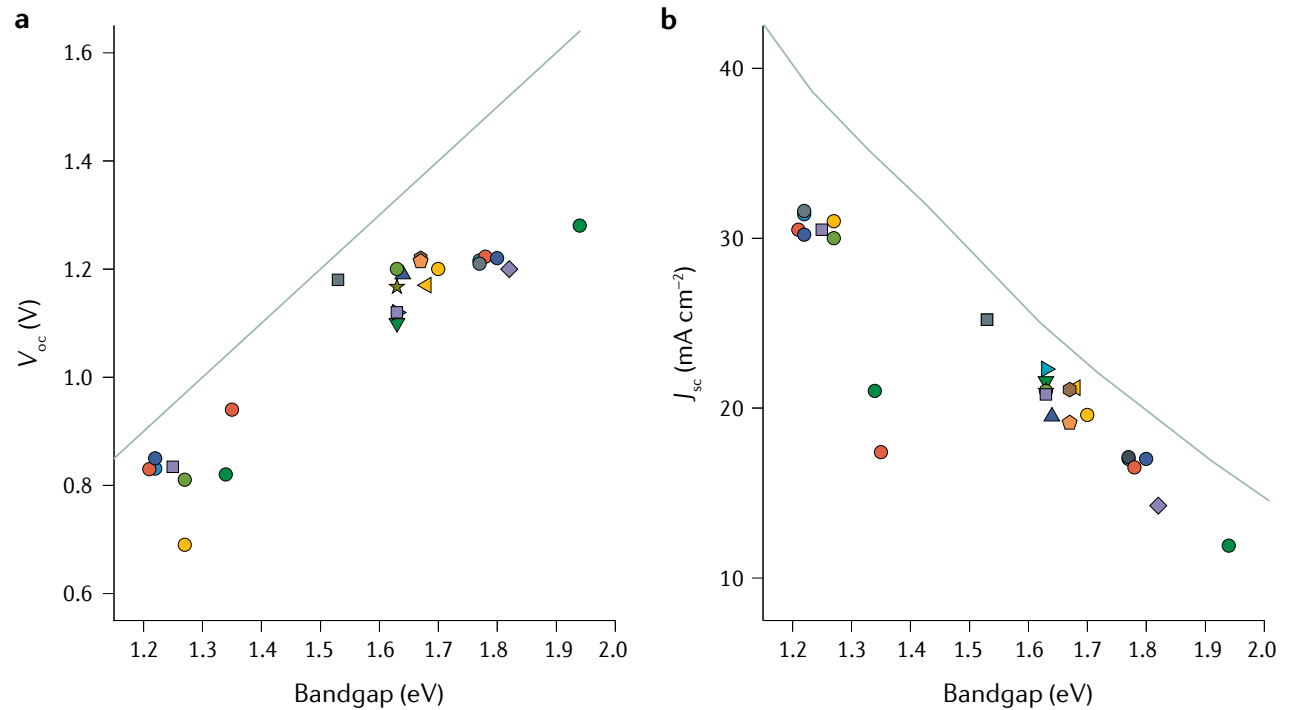

\begin{tabular}{|c|}
\hline 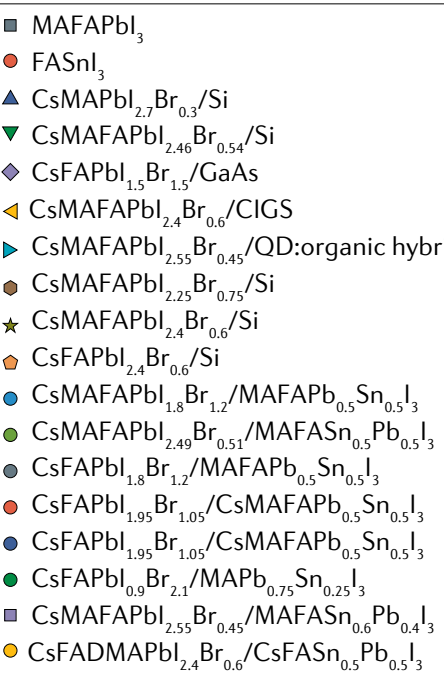 \\
\hline
\end{tabular}

Fig. 1 | State-of-the-art tandem subcells based on wide-bandgap and narrow-bandgap MHPs. a | Summary of the open-circuit voltage $\left(V_{o c}\right)$ as a function of the bandgap for various materials ${ }^{3-18} \cdot V_{o c}$ loss is the main limiting factor for the photovoltaic performance of wide-bandgap metal halide perovskite (MHP) subcells. b | Summary of the short-circuit current $\left(V_{s c}\right)$ as a function of the bandgap. $J_{s c}$ loss is the main limiting factor for the photovoltaic performance of narrowbandgap MHP subcells ${ }^{3-18}$. The $V_{\text {oc }}$ and $J_{\text {sc }}$ of MAFAPbl ${ }_{3}\left(\right.$ REF. $^{19}$ ) and FASnl $\left(\right.$ REF. $^{22}$ ) solar cells are included for reference. CIGS, copper indium gallium (di)selenide; DMA, dimethylammonium; FA, formamidinium; MA, methylammonium; QD, quantum dot.

It is thus clear that the full potential of WB MHPs and NB MHPs has not yet been reached. In this Review, we critically assess the unique physical/chemical activity of defects that may lead to energy loss and instability in both WB MHPs and NB MHPs, by summarizing the most recent findings and strategies possibly leading to the realization of highly efficient and defect-tolerant materials.

\section{Wide-bandgap metal halide perovskites Origin of halide segregation}

Thermodynamics of halide segregation. In 2015 , Michael McGehee and co-workers demonstrated the occurrence of photoinduced halide segregation in mixed halide $\mathrm{MAPb}\left(\mathrm{I}_{1-x} \mathrm{Br}_{x}\right)_{3}$ perovskites with variable bromide content $^{20}$. A clear signature of the creation of regions rich in bromide and iodide upon light soaking was the appearance of a low-energy emission in the photoluminescence (PL) spectrum corresponding to the I-rich phase. It was later found that photoinduced halide segregation is a universal phenomenon in $3 \mathrm{D}$ mixed $\mathrm{I}-\mathrm{Br}$ $\mathrm{MHP}^{20,23,24}$. Different thermodynamic models have been proposed by several groups to predict the formation of a miscibility gap in the free-energy curve of the perovskite solid solution under illumination ${ }^{25-28}$. Aron Walsh and co-workers suggested that intense illumination helps in overcoming the kinetic barrier of phase demixing by locally changing the temperature ${ }^{25}$. However, there is experimental evidence for a remixing rather than a demixing of halides on heating and photoheating ${ }^{29-32}$. Naomi Ginsberg and collaborators suggested that charged excitations due to strong electron-phonon coupling may generate lattice strain sufficient to demix the solid solution and that such a process is mainly correlated to the polarity of the A-site cation ${ }^{26,33}$. The electron-phonon coupling model was further extended by Udo Bach and collaborators, who claimed that a sufficiently high photon flux can induce long-range saturation of the lattice with polarons and deform the lattice uniformly, cancelling the lattice strain gradient for driving halide segregation and thus leading to halide remixing ${ }^{34}$. This mechanism is still under debate, and the main objections concern the lack of experimental evidence about the difference in electron-phonon coupling when changing the cation ${ }^{35}$, and, most notably, the fact that carrier lifetimes are too short with respect to ion dynamics to trigger long-standing, albeit reversible, material transformations.

Ion migration and defects. Halide segregation in $\mathrm{WB}$ MHPs requires the migration of halide ions, and it is most likely a defect-driven process. Among the different MHP constituents, halides are the most mobile species; indeed, very low activation energies of $0.1-0.6 \mathrm{eV}$ $\left(\mathrm{REFS}^{36-39}\right)$ were reported for both iodine vacancy $\left(\mathrm{V}_{\mathrm{I}}^{+}\right)$ and interstitial $\left(\mathrm{I}_{i}^{-}\right)$migration (FIG. 2a). The reported migration barriers for bromine vacancy $\left(\mathrm{V}_{\mathrm{Br}}^{+}\right)$and interstitial $\left(\mathrm{Br}_{\mathrm{i}}^{-}\right)$defects are around $0.3 \mathrm{eV}$ and are very close to those of their iodine counterparts ${ }^{40}$. There are two kinds of neutral intrinsic disorder reactions that might produce halide vacancies $\left(\mathrm{V}_{\mathrm{X}}{ }^{+}, \mathrm{X}=\mathrm{I}\right.$ or $\left.\mathrm{Br}\right)$ and interstitials $\left(\mathrm{X}_{\mathrm{i}}^{-}\right)$in $\mathrm{APbI}_{3}$, Schottky $^{40}$ and Frenkel disorder ${ }^{41}$ :

$$
\begin{aligned}
& \mathrm{Pb}_{\mathrm{Pb}}+2 \mathrm{X}_{\mathrm{X}} \rightarrow \mathrm{V}_{\mathrm{Pb}}^{2-}+2 \mathrm{~V}_{\mathrm{X}}^{+}+\mathrm{PbX}_{2} \\
& \mathrm{~A}_{\mathrm{A}}+\mathrm{X}_{\mathrm{X}} \rightarrow \mathrm{V}_{\mathrm{A}}^{-}+\mathrm{V}_{\mathrm{X}}^{+}+\mathrm{AX} \\
& \mathrm{X}_{\mathrm{X}} \rightarrow \mathrm{V}_{\mathrm{X}}^{+}+\mathrm{X}_{\mathrm{i}}^{-}
\end{aligned}
$$



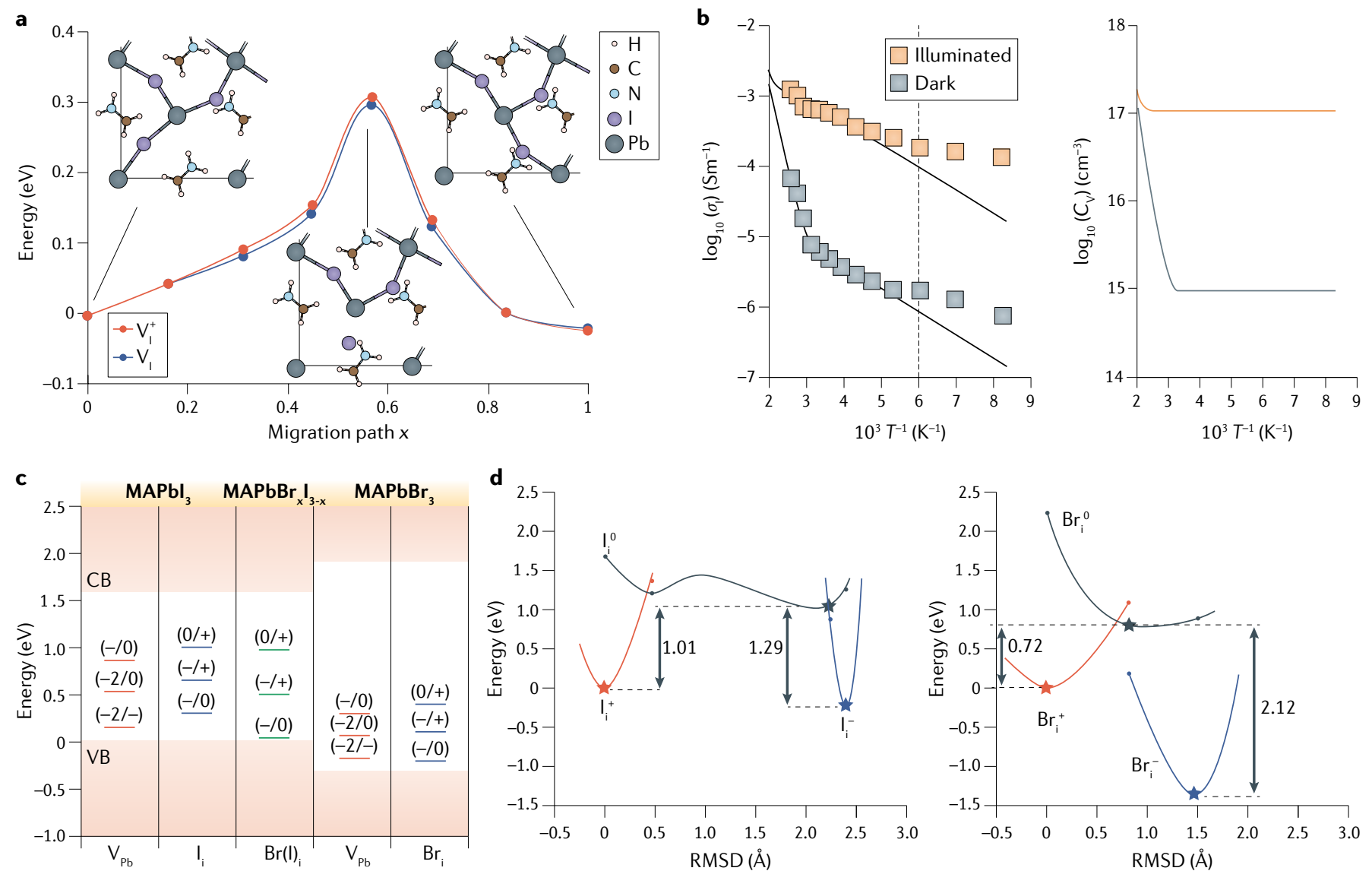

Fig. 2 | Impact of point defects on halide segregation. a | Energy profiles along the migration path of an iodide vacancy in $\mathrm{MAPbl}_{3}$. The path of $\mathrm{V}_{1}$ corresponds to the neutral system, whereas that of $\mathrm{V}_{1}^{+}$reflects a system in which one electron is removed from the calculation supercell. The insets show the structures of the nudged elastic-band images at the initial, transition and final states. $\mathbf{b}$ | The left plot shows the enhancement of ionic conductivity $\left(\sigma_{1}\right)$ in $\mathrm{MAPb}_{3}$ under illumination, which is mainly due to the increase in iodide vacancy concentration ( $C_{\boldsymbol{V}}$, right plot). $\mathbf{c} \mid$ Transition energies of stable hole-trapping defects in $\mathrm{MAPbl}_{3}, \mathrm{MAPbBr}_{3}$ and $\mathrm{MAPbBr} \mathrm{I}_{3-x}$. Hole trapping through these defects produces halide vacancies. The energetics of trap states are mainly contributed by iodine, even in mixed I-Br perovskites, whereby the $(-/ 0)$ transition of the mixed $\mathrm{I} / \mathrm{Br}$ interstitial defect $\mathrm{Br}(\mathrm{I})_{\mathrm{i}}$ (which is the transition from a negative to a neutral defect via hole trapping) remains almost constant as the valence band deepens. $\mathbf{d}$ | Configuration coordinate diagrams representing the relative energies of iodine and bromine interstitials, indicating that the negative bromine interstitial is more difficult to oxidize than its iodine counterpart. CB, conduction band; RMSD, root-mean-square deviation; $\mathrm{VB}$, valence band; $\mathrm{V}_{\mathrm{Pb}}$, Pb vacancy; $\mathrm{X}_{\mathrm{i}}$, interstitial $\mathrm{X}$. Panel $\mathbf{a}$ adapted with permission from $\mathrm{REF} .{ }^{38}$, ACS. Panel $\mathbf{b}$ adapted with permission from REF. ${ }^{41}$, RSC. Panel $\mathbf{c}$ reprinted with permission from REF. ${ }^{43}$, ACS. Panel $\mathbf{d}$ adapted with permission from REF. ${ }^{42}$, Wiley.

where $\mathrm{Pb}_{\mathrm{Pb}}, \mathrm{A}_{\mathrm{A}}$ and $\mathrm{X}_{\mathrm{X}}$ represent the atom or molecule staying at its own site in the perovskite lattice. All these reactions produce halide vacancies $\left(\mathrm{V}_{\mathrm{x}}^{+}\right)$, whereas only Eq. (3) can generate halide interstitials $\left(\mathrm{X}_{\mathrm{i}}^{-}\right)$in nearly stoichiometric conditions of growth, indicating that $\mathrm{V}_{\mathrm{x}}^{+}$ are potentially present in higher concentration than $\mathrm{X}_{\mathrm{i}}^{-}$ in the perovskite.

The ionic conductivity in MHPs is commonly seen to be enhanced under illumination. Denis Barboni and Roger De Souza ${ }^{41}$ found that this phenomenon can be reproduced by simply assuming that illumination generates a large number of $\mathrm{V}_{\mathrm{I}}^{+}$(FIG. 2b). They suggested that these $\mathrm{V}_{\mathrm{I}}^{+}$are generated indirectly by first neutralizing $\mathrm{I}_{\mathrm{i}}^{-}$by photogenerated holes

$$
\mathrm{X}_{\mathrm{i}}^{-}+\mathrm{h}^{+} \rightarrow \mathrm{X}_{\mathrm{i}}^{0}
$$

and then shifting the Frenkel process (Eq. (3)) to the right. The process described in Eq. (4) should be feasible, because the electronic levels for iodine and bromine in the energy-band diagram are predicted to be $0.29 \mathrm{eV}$ and $0.12 \mathrm{eV}$ above the valence band edge, respectively ${ }^{42}$. The direct formation of $\mathrm{V}_{\mathrm{I}}^{+}$is not favoured because it requires suitable amounts of energy and momentum, and photons possess negligible momentum. Experimentally, there is evidence that both excess holes ${ }^{43-45}$ and electrons ${ }^{46}$ aid halide migration. This controversial experimental observation may be reconciled by considering the amphoteric nature of halide interstitial defects. The formation of both negative and positive halide interstitials $\left(\mathrm{X}_{\mathrm{i}}^{-}\right.$and $\mathrm{X}_{\mathrm{i}}^{+}$) is energetically favourable ${ }^{42,47}$ (FIG. 2c,d). These species can trap holes and electrons, respectively, leading to the formation of the metastable neutral interstitial $\mathrm{X}_{\mathrm{i}}^{0}$. The migration of such species causes the formation 
of $\mathrm{X}_{2}$ through a bimolecular process, whose efficiency depends on the density of the interstitial defects and/or on the lifetime of $\mathrm{X}_{\mathrm{i}}^{0}$ species ${ }^{48}$. The loss of $\mathrm{X}_{2}$ unbalances Eq. (3) and boosts the formation of mobile defects. Thus, both electron and hole trapping can increase ion conductivity. Importantly, the $\mathrm{X}_{\mathrm{i}}^{0}$ defect formed through electron trapping at $\mathrm{X}_{\mathrm{i}}^{+}$has a longer lifetime than those formed upon hole trapping and favours the formation of $\mathrm{X}_{2}$. Nevertheless, a higher density of hole-trapping states may compensate the contribution of hole trapping to this bimolecular event.

Regarding the nature of the halide, iodine and bromine defects may contribute differently to halide migration in the mixed halide perovskite. First, density functional theory (DFT) calculations suggest a lower defect formation energy for $\mathrm{V}_{\mathrm{Br}}{ }^{+}$than for $\mathrm{V}_{\mathrm{I}}^{+}$; second, $\mathrm{I}_{\mathrm{i}}^{-}$is easier to oxidize than $\mathrm{Br}_{\mathrm{i}}^{-}$in the mixed halide perovskite through hole trapping owing to the lower electronegativity of iodine, and thus illumination should preferentially increase the formation of iodide vacancies and consequently the conductivity of iodide ${ }^{42,43}$ (FIG. 2c,d). In addition, it was suggested ${ }^{49}$ that $\mathrm{I}^{-}$and $\mathrm{Br}^{-}$possibly show different hopping rate under illumination, as $\mathrm{Br}^{-}$ vacancy migration features a lower activation energy than $\mathrm{I}^{-}$vacancy in mixed I-Br MHPs. The different concentration and hopping rate of iodine and bromine defects are possible causes of halide segregation in WB MHPs, where they may generate a gradient of I-to- $\mathrm{Br}$ ratio.

A-site cations with different polarity may interact differently with migrating halide defects. Edoardo Mosconi and Filippo De Angelis used ab initio molecular dynamic simulations to investigate the migration of $\mathrm{V}_{\mathrm{I}}^{+}$coupled to thermal disorder ${ }^{50}$. They found that the $\mathrm{MA}^{+}$orientation dynamic facilitates iodine migration. Therefore, the use of less polar and less orientationally mobile A-cations (such as $\mathrm{FA}^{+}$and/or $\mathrm{Cs}^{+}$) may slow halide migration in MHPs, possibly leading to more stable optoelectronic devices. The beneficial effect of $\mathrm{MA}^{+}$replacement is supported by the suppression of halide segregation in WB MHPs based on Cs-FA (REF. ${ }^{23}$ ) and pure Cs (REF. ${ }^{24}$ ), compared with MA-based WB MHPs.

Grain boundaries and surface defects. The presence of crystal terminations, including grain boundaries and surfaces, initiates the formation of the I-rich phase during halide segregation, as evidenced by luminescence mapping of both monocrystalline ${ }^{51,52}$ and polycrystalline ${ }^{26,53}$ WB MHPs. Using $\mathrm{MAPbX}_{3}$ as a model system, combined scanning tunnelling microscopy experiments and DFT calculations showed that the (001) surface is the most common and thermodynamically stable crystal surface ${ }^{54-56}$, and it can be $\mathrm{PbI}_{2}$ - or MAI-terminated ${ }^{57,58}$. By contrast, $\mathrm{MAPbI}_{3}$ monolayers deposited in ultrahigh vacuum ${ }^{59}$ and vacuum-cleaved $\mathrm{MAPbBr}_{3}$ single crystals $^{54}$ show MAX-terminated surfaces. However, the fabrication of highly crystallized MHP thin films requires annealing at temperatures up to $100^{\circ} \mathrm{C}$, which can make the volatile organic halide species evaporate from the surface, leaving a $\mathrm{PbX}_{2}$ termination. A study of the formation and migration of $\mathrm{I}_{\mathrm{i}}^{-} / \mathrm{V}_{\mathrm{I}}^{+}$Frenkel pairs on $\mathrm{MAPbI}_{3}$ surfaces by DFT calculations demonstrated that the formation energy of these defects can be greatly reduced, from $0.86 \mathrm{eV}$ to $0.03 \mathrm{eV}$, by switching from an MAI-terminated to a $\mathrm{PbI}_{2}$-terminated surface ${ }^{58}$ (FIG. 3a). The results accord well with experimental observations, suggesting that halide migration in lead halide perovskites is dominated by surfaces and grain boundaries ${ }^{60,61}$.

Several models have been proposed to link surfaces and grain boundaries with photoinduced halide segregation. Samuel Stranks and co-workers suggested that electron trapping at the surface generates an electric field that drives iodine away from the $\mathrm{MAPbI}_{3}$ surface $^{62}$. Richard Friend and collaborators took into account the higher hopping rate of bromide with respect to iodide along the vertical direction, postulating that it results in the formation of an I-rich phase on the surface ${ }^{49}$. Michael McGehee and co-workers suggested that halide segregation is driven by surface electron trapping ${ }^{45}$ and proposed that iodide may have a higher migration rate owing to the preferential increase in the formation of iodine vacancies with respect to bromine vacancies under photoexcitation. However, this hypothesis contradicts the observation of the formation of an I-rich phase at grain boundaries. Laura Herz and co-workers found that the lower the charge-carrier density, that is, the closer to the defect density, the higher the efficacy of a charge carrier in inducing halide segregation (per unit carrier $^{63}$ (FIG. 3b). They also identified electron trapping as the main trapping process, where the trapping sites are assumed to be randomly distributed rather than limited to the surface. These researchers also suggested that such electron traps should be neutrally charged and located near the grain boundaries within the crystal lattice distortions ${ }^{64}$. Further experimental evidence of the fact that surface electron trapping aids halide segregation, as shown schematically in FIG. 3c, was provided by Jeffrey DuBose and Prashant Kamat ${ }^{44}$.

Although the above studies consistently suggest that electron trapping drives halide segregation, none of them have clarified the origin of this process and the nature of the trap states. It was demonstrated that accumulation of positive space charges at grain boundaries induces halide segregation owing to preferential drift of iodine towards grain boundaries to form an I-rich phase $^{53}$ (FIG. 3 d). These positive space charges exist before light illumination, indicating that their formation is not due to hole funnelling under illumination but to hole trapping. Surface hole trapping is highly possible, as the formation energy of $\mathrm{I}_{\mathrm{i}}^{-} / \mathrm{V}_{\mathrm{I}}^{+}$Frenkel pairs on the surface is very $l o w^{58}$. Besides, the concentration of $\mathrm{I}_{\mathrm{i}}^{-}$defects at grain boundaries increases substantially under illumination ${ }^{42}$, enhancing their hole-trapping capability. It was demonstrated ${ }^{48}$ that polyethylene oxide, which passivates undercoordinated $\mathrm{Pb}$ sites ${ }^{65}$, hampers the photoinduced formation of mobile defects in perovskites. The passivated sites are those where $\mathrm{I}_{2}$ molecules, created upon electron or hole trapping as discussed in the previous paragraph, migrate upon photoexcitation, triggering the formation of further defects (FIG. 3e). This knowledge, based on the study of $\mathrm{MAPbI}_{3}$ thin films, can be transferred to mixed halide perovskites and help to rationalize the role of surfaces and defects in influencing ion migration in these systems. 

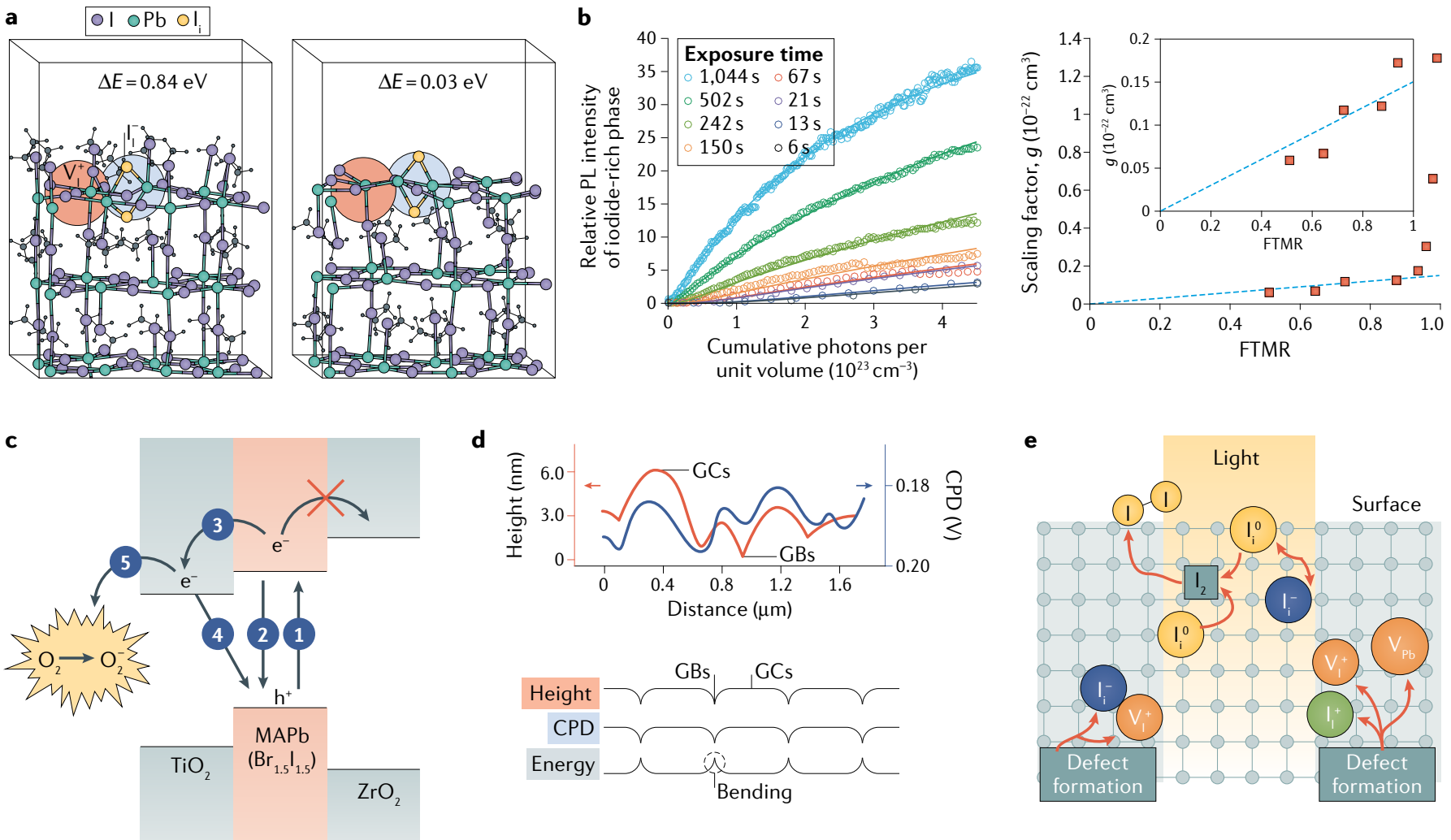

Fig. 3 | Role of GBs in halide segregation. a | Optimized structures and formation energies $(\Delta E)$ for an negative I interstitial $\left(\mathrm{I}_{\mathrm{i}}^{-}\right) / \mathrm{I}$ vacancy $\left(\mathrm{V}_{1}^{+}\right)$ Frenkel pair at MAl-terminated (left) and $\mathrm{Pbl}_{2}$-terminated (right) surfaces. b | Evolution of the photoluminescence (PL) intensity from the low-bandgap I-rich phase of $\mathrm{MAPb}\left(\mathrm{Br}_{0.5} \mathrm{I}_{0.5}\right)_{3}$ plotted against the number of incident photons per unit volume for different overall exposure times to illumination but constant total incident photons (left). Solid lines are a fit based on a fitting parameter, $g$, whose evolution as a function of the fraction of trap-mediated recombination (FTMR) is shown on the right graph. The parameter $g$ can be thought of as the charge-carrier efficacy for inducing halide segregation. The excited charge carriers that recombine through trap states mediate halide segregation. $\mathbf{c}$ | Extraction of electrons from an excited metal halide perovskite (MHP) into the electron-transporting layer $\left(\mathrm{TiO}_{2}\right)$, followed by trapping of electrons by $\mathrm{O}_{2}$, causes hole accumulation in the MHP film and thus assists halide segregation. $\mathbf{d}$ | Topology profile and corresponding contact potential difference (CPD) profile of $\mathrm{MAPbBr}_{0.9} \mathrm{I}_{2.1}$ measured by atomic force microscopy (top). These two profiles show surface potential down-bending at the grain boundary (GB) and thus energy up-bending there (bottom), which is indicative of a higher concentration of positive space charge near the grain boundary. Under illumination, the excess positive space charges close to the grain boundaries help to drive the migration of negative iodine ions towards grain boundaries. e| Schematic showing the role of both electron and hole trapping in forming $\mathrm{I}_{0}$ species. GC, grain centre; MA, methylammonium. Panel a reprinted with permission from REF. ${ }^{58}$, ACS. Panel $\mathbf{b}$ reprinted with permission from REF. ${ }^{63}$, CC BY 4.0 (https://creativecommons.org/licenses/by/4.0/). Panel c adapted with permission from REF. ${ }^{44}$, ACS. Panel $\mathbf{d}$ adapted with permission from REF. $^{53}$, ACS. Panel e adapted from REF. ${ }^{48}$, Springer Nature Limited.

\section{Origin of energy losses}

In a typical semiconductor, one would expect the solar cell $V_{\text {oc }}$ to scale with the absorber bandgap. By contrast, in MHPs the $V_{\text {oc }}$ shows a plateau in the bandgap range 1.7-2.2 eV, which largely limits the PCE of solar cells based on MHPs with bandgap in this range. Many reports have attributed this $V_{\text {oc }}$ plateau to the photoinduced halide segregation mentioned above. However, the reported $V_{\text {oc }}$ values are extracted by current-voltage $(I-V)$ scans performed in tens of seconds, in which the $V_{\text {oc }}$ shows barely any discrepancy between forward and backward scans. This indicates that photoinduced phase segregation has not kicked in yet to substantially affect the $V_{\text {oc }}$ during the time of the $I-V$ scan. The initial $V_{\text {oc }}$ of solar cells based on WB MHPs may be determined by the quality of pristine thin films and the device configuration, including possibly inadequate selective contacts, whereas the impact of photoinduced halide segregation on the $V_{\text {oc }}$ most likely shows up under long-term operation in conditions of continuous illumination.
Bulk traps. Early studies of defect physics in MHPs revealed that point defects with low formation energy have energy levels within, or very close to, the conduction band or valence band, forming relatively shallow traps ${ }^{66}$. This unusual defect physics in MHPs is considered to be the origin of their defect-tolerant nature. Nevertheless, the presence of deep trap states is experimentally tangible. For instance, the photoluminescence quantum yield (PLQY) has a strong dependence on the excitation density, which implies the presence of trap-filling processes mediated by fairly deep states ${ }^{67,68}$. The intrinsic soft nature of MHPs indicates that the trap density might change substantially as these electronic trap states are created or annihilated under external stimuli such as light or heat.

DFT calculations showed that $\mathrm{MA}_{\mathrm{i}}, \mathrm{X}_{\mathrm{i}}, \mathrm{V}_{\mathrm{Pb}}$ and $\mathrm{V}_{\mathrm{x}}$ are the most thermodynamically stable defects ${ }^{42}$. Among these defects, those that can introduce deep energy levels within the bandgap are the halide interstitials $\mathrm{X}_{\mathrm{i}}$ and $\mathrm{V}_{\mathrm{Pb}}$. Negative halide interstitials are not stable for Fermi levels close to the valence band maximum (VBM), where 
they tend to be oxidized to the corresponding positive $\mathrm{X}_{\mathrm{i}}^{+}$interstitials ${ }^{69}$. In addition, $\mathrm{I}_{\mathrm{i}}^{+}$are only moderately stable at the intrinsic Fermi level (close to midgap in 1.6-eV bandgap MHPs) and show densities comparable to those of negative $\mathrm{I}_{\mathrm{i}}^{-}$. By contrast, the higher defect formation energy of $\mathrm{Br}_{\mathrm{i}}^{+}$results in its negligible density in $\mathrm{MAPbBr}_{3}$. The higher stability of positive iodine interstitials is mainly due to the lower electronegativity of iodine compared with bromine, in accordance with the higher stability of $\mathrm{I}_{3}^{-}$compared with $\mathrm{Br}_{3}{ }^{-}$. $\mathrm{V}_{\mathrm{Pb}}{ }^{2-}$ also has a fairly low defect formation energy in $\mathrm{MAPbI}_{3}$, comparable to that of $\mathrm{I}_{\mathrm{i}}^{-}$, and thus a comparable defect density. Like $\mathrm{I}^{-}$, $\mathrm{V}_{\mathrm{Pb}}{ }^{2-}$ tends to act as a hole trap and spontaneously lead to the formation of the $\mathrm{I}_{3}{ }^{-}$trimer for Fermi levels close to the VBM, that is, in p-doped perovskites ${ }^{47}$.

Negative and positive halide interstitials are active in trapping holes and electrons, respectively. However, electron trapping by $\mathrm{X}_{\mathrm{i}}^{+}$creates a large effective barrier $(\sim 0.3 \mathrm{eV})$ to charge recombination owing to the large lattice reorganization. This recombination barrier is a possible reason for the extremely long lifetimes of filled electron traps. By contrast, hole trapping by negative interstitials induces a smaller lattice rearrangement and results in a smaller recombination energy barrier, and thus short lifetime of the trapped species ${ }^{42}$. As a result, the long-lived electron trapping is associated with less effective loss channels than short-lived hole trapping, explaining the impressive defect tolerance found in MHPs with bandgap below $1.7 \mathrm{eV}\left(\mathrm{REF}^{70}\right)$. However, at higher $\mathrm{Br}$ contents, a reduction of the $\mathrm{X}_{\mathrm{i}}^{+}$ density is expected to occur, owing to the high defect formation energy of $\mathrm{Br}_{i}^{+}$and thus its negligible contribution to the overall $\mathrm{X}_{\mathrm{i}}^{+}$density. Therefore, short-lived hole trapping may become prominent for larger bandgaps obtained by increasing the $\mathrm{Br}$ content in mixed halide MHPs, and it may thus contribute to the larger $V_{\text {oc }}$ loss in WB MHPs. Besides halide traps, Laura Herz and collaborators observed the existence of positively charged traps, as they found increased emitted PL intensity from the $\mathrm{MAPbI}_{1.5} \mathrm{Br}_{1.5}$ perovskite when the overall electric field was directed away from the illuminated side of the device $^{64}$ (FIG. 4a). They proposed that these traps could be interstitial $\mathrm{MA}_{\mathrm{i}}^{+}$ions in the perovskite crystal. However, calculations predict that $\mathrm{MA}_{\mathrm{i}}^{+}$is not active in trapping because its energy level lies within the conduction band, so further investigation is probably necessary to clarify the involvement of $\mathrm{MA}_{\mathrm{i}}^{+}$in causing energy losses ${ }^{42}$.

Grain boundaries and surface traps. Grain boundaries and surfaces of polycrystalline MHPs tend to have high trap density - as evidenced by the orders-of-magnitude smaller trap density measured inside single-crystalline MHPs compared with that at their edges (FIC. 4b) - and cause severe $V_{\text {oc }} \operatorname{loss}^{71}$ (FIG. 4c). Many works have demonstrated that introducing a $\mathrm{PbX}_{2}$-terminated surface is very helpful to achieve high efficiency in solar cells based on MHPs with mid-range bandgap $(<1.7 \mathrm{eV})^{72,73}$. A comparison of $\mathrm{PbI}_{2}$-excess and $\mathrm{PbI}_{2}$-deficient $\mathrm{MHP}$ thin films showed that $\mathrm{PbI}_{2}$-deficient devices present lower efficiency, mainly owing to limited $J_{\text {sc }}$, which was attributed to poor charge extraction caused by the enriched organic species at the grain boundaries ${ }^{74}$. Lower $V_{\text {oc }}$ loss was also observed in $\mathrm{PbI}_{2}$-deficient devices than in $\mathrm{PbI}_{2}$ excess devices, which is due to suppressed non-radiative recombination, as indicated by higher PL and external electroluminescence efficiency $\left(\mathrm{EQE}_{\mathrm{EL}}\right)$.

Beyond photovoltaic applications, the use of excess AX has attracted much attention and achieved great success in both near-infrared and green light-emitting diodes (LEDs), which reached $\mathrm{EQE}_{\mathrm{EL}}$ over $20 \%{ }^{75-79}$. The authors of such works revealed that the metallic lead in MHPs acts as a non-radiative trap that is very detrimental to $\mathrm{EQE}_{\mathrm{EL}}{ }^{75}$. By introducing excess $\mathrm{AX}$, the amount of metallic $\mathrm{Pb}$ is reduced, offering a substantial improvement in PLQY (FIG. 4d) and EQE $\mathrm{EL}_{\mathrm{EL}}$. Through combined DFT calculations and photophysical characterizations, it was shown that the PL intensity decrease in MHPs is possibly related to the formation and stabilization of $\mathrm{I}_{2}$ on the surface, which is accompanied by the formation of metallic $\mathrm{Pb}\left(\mathrm{REF}^{48}{ }^{48}\right.$ (FIG. 3e). The reaction may involve the formation of Frenkel pairs of $\mathrm{V}_{\mathrm{I}}^{+}$and $\mathrm{I}_{i}^{-}$, which is thermodynamically favoured on MAI-deficient ( $\mathrm{PbI}_{2}$-rich) surfaces ${ }^{58}$. The formation of metallic $\mathrm{Pb}$ and $\mathrm{I}_{2}$ and its correlation with the existence of $\mathrm{PbI}_{2}$-rich regions in MHPs (FIG. 4e) have been confirmed ${ }^{80,81}$. Based on this evidence, the beneficial effect of AX excess in improving the $V_{\text {oc }}$ is due to the reduced formation of metallic $\mathrm{Pb}$ and $\mathrm{I}_{2}$ and decreased non-radiative recombination. On increasing the bromine content in mixed halide MHPs, the surface tends to be prevalently $\mathrm{PbX}_{2}$-terminated, owing to the increase of organic bromide species such as $\mathrm{MABr}$ or $\mathrm{FABr}$ that are more volatile than their iodine counterparts $^{82}$. Therefore, non-radiative recombination due to the formation of metallic $\mathrm{Pb}$ becomes even more prominent, which may represent one of the major $V_{\text {oc }}$ loss mechanisms in solar cells based on WB MHPs.

Halide segregation. Halide segregation can contribute to $V_{\mathrm{oc}}$ loss owing to the funnelling of photocarriers to the I-rich region, which has lower bandgap and lower bandedge energies. Nanoscale composition mapping revealed intrinsic halide segregation - that is, in pristine films, before illumination - in solution-processed mixed halide perovskites ${ }^{83,84}$. This segregation possibly originates from the difference between the solubility of iodide and bromide species, resulting in heterogenous nucleation during thin-film deposition. Besides intrinsic halide segregation, WB MHPs undergo extra halide segregation under light illumination and electrical fields, which leads to $V_{\text {oc }}$ loss during the operation of solar cells. Henry Snaith and collaborators investigated the origin of the $V_{\text {oc }}$ loss in solar cells based on WB MHPs ${ }^{85}$ (FIG. 5a,b). In addition to the loss in the radiative $V_{\text {oc }} \operatorname{limit}\left(V_{\text {oc,rad }}\right)$, they proposed that charge-carrier funnelling due to halide segregation could reduce non-radiative loss and compensate $V_{\text {oc,rad }}$. Interestingly, their modelling results demonstrated that non-radiative recombination, rather than halide segregation, dominates $V_{\text {oc }}$ loss in solar cells based on WB MHPs. However, the influence of halide segregation on $V_{\text {oc }}$ may be underestimated without considering the distribution of the I-rich phase. Moreover, it was suggested that the $V_{\text {oc }}$ loss in WB MHP solar cells is mainly caused by the comparatively high interfacial defect density and energy level mismatch ${ }^{86}$, rather than byI-rich domains. 


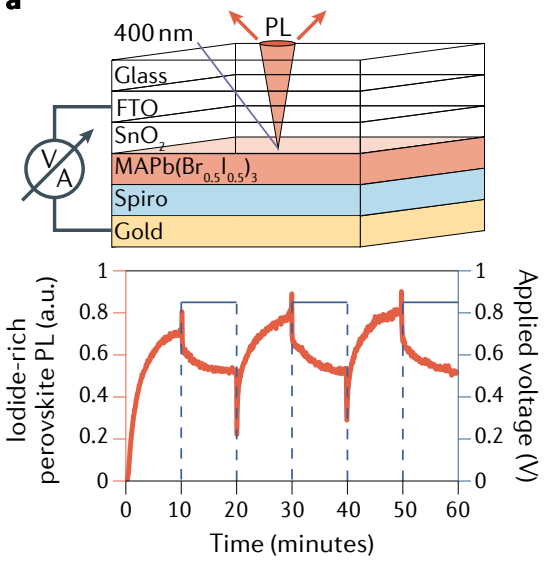

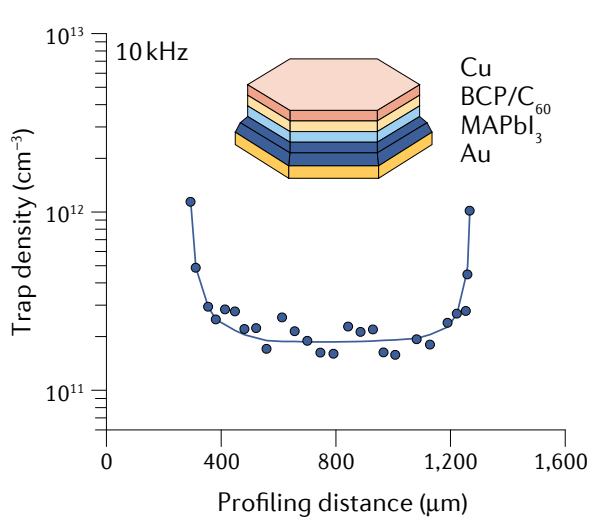

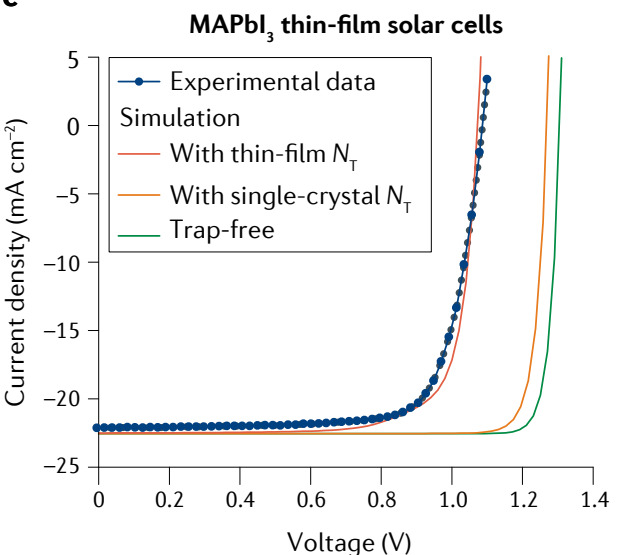

d

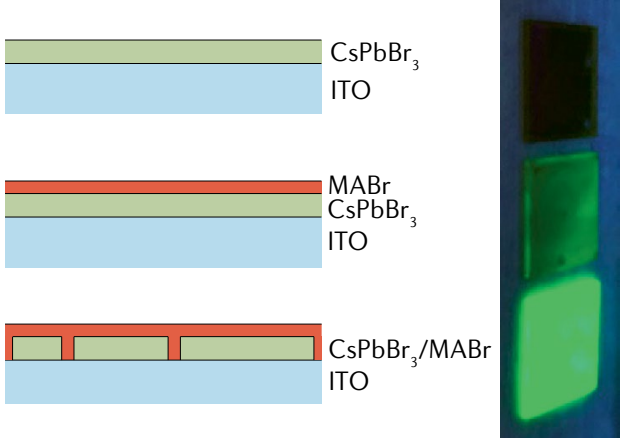

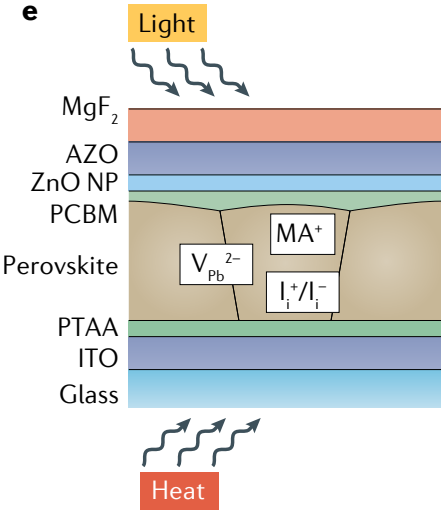
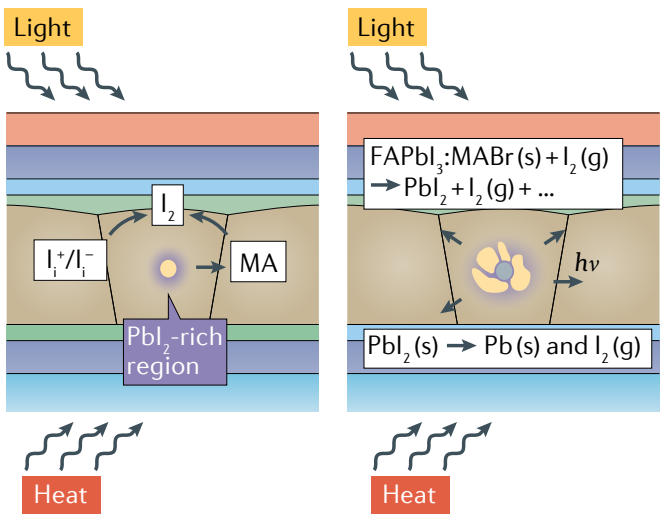

$\mathrm{Pb} \quad \square \mathrm{Pbl}_{2} \quad \square \mathrm{I}_{2}$ and other volatile degradation compounds
Fig. 4 | Trap-mediated performance and stability of perovskite optoelectronic devices. a | Behaviour of the photoluminescence (PL) originating from the low-bandgap I-rich phase of a mixed halide perovskite device as a changing voltage (plotted in blue) is applied under continuous-wave illumination $\left(400 \mathrm{~nm}, 110 \mathrm{~mW} \mathrm{~cm}^{-2}\right)$. The PL enhancement at $0 \mathrm{~V}$ and its quenching at $0.85 \mathrm{~V}$ over time indicate the existence of some kind of positive mobile electron trap, which might be $\mathrm{MA}^{+} . \mathbf{b}$ | Dependence of the trap density on the profiling distance of a $\mathrm{MAPbl}_{3}$ single crystal measured by drive-level capacitance profiling. The inset shows the device structure. $\mathbf{c} \mid$ Measured and simulated curves of current density versus voltage $(U-V)$ for planarstructured solar cells based on $\mathrm{MAPbl}_{3}$ polycrystalline thin films. For the simulation, the bulk density is fixed at $5.0 \times 10^{14} \mathrm{~cm}^{-3}$. The interface trap density is changed from $1.0 \times 10^{17} \mathrm{~cm}^{-3}$ (common interface trap density of polycrystalline $\mathrm{MAPbl}_{3}$ thin film) to $2.0 \times 10^{15} \mathrm{~cm}^{-3}$ (common bulk trap density of a $\mathrm{MAPbl}_{3}$ single crystal), and the corresponding simulated $J-V$ curves are shown in red and orange, respectively. The $J-V$ curve simulated by assuming the absence of traps is shown for comparison (green). $N_{\tau}$ in the overall trap density at the interface and in the bulk. $\mathbf{d}$ /Schematic illustration of $\mathrm{CsPbBr}_{3}$ perovskite devices in which the introduction of excess $\mathrm{MABr}$ on the top surface and at grain boundaries reduces non-radiative traps. The photographs of the corresponding three as-prepared perovskite films are taken under ultraviolet light. e | Schematic illustration of a $\mathrm{Pbl}_{2}$-rich region inducing the formation of metallic $\mathrm{Pb}$ and $\mathrm{I}_{2}$, which is detrimental to the device operational stability and performance. AZO, Al-doped $\mathrm{ZnO}$; BCP, bathocuproine; FTO, fluorine-doped tin oxide; ITO, indium tin oxide; MA, methylammonium; NP, nanoparticle; PCBM, [6,6]-phenyl- $\mathrm{C}_{61}$-butyric acid methyl ester; PTAA, poly[bis(4-phenyl)(2,4,6-trimethylphenyl)amine]. Panel $\mathbf{a}$ adapted with permission from REF. ${ }^{64}$, Wiley. Panels $\mathbf{b}$ and $\mathbf{c}$ reprinted with permission from REF. ${ }^{71}$, AAAS. Panel $\mathbf{d}$ adapted from REF. ${ }^{77}$, Springer Nature Limited.
Besides $V_{\text {oc }}$ loss, Laura Herz and co-workers showed that photoinduced phase segregation can lead to a marked loss in EQE above the absorption edge of WB MHPs, which is due to the funnelling of photocarriers from the mixed phase to the I-rich phase, where the current extraction efficiency is lower ${ }^{64}$ (FIG. 5c). They also showed that a small fraction of current can be extracted from the I-rich phase, implying that the I-rich phase preferentially forms around grain boundaries and surfaces.

Energy-level alignment. With increasing $\mathrm{Br}$ content in mixed halide MHPs, the bandgap is enlarged, and the conduction and valence band levels shift downward and upward, respectively. It must be noted that the commonly used electron transport layers (ETLs) and hole transport layers (HTLs) are optimized for $\mathrm{MAPbI}_{3}$. As a matter of fact, the energy level can vary by $\sim 0.6 \mathrm{eV}$ in conduction band minimum (CBM) and by $\sim 0.8 \mathrm{eV}$ in $\mathrm{VBM}$ by mixing cations $\left(\mathrm{Cs}^{+}, \mathrm{MA}^{+}\right.$and $\left.\mathrm{FA}^{+}\right)$at A-site and halides (I and $\mathrm{Br}$ ) at X-site in the MHP lattice ${ }^{87}$. Therefore, designing ETLs and HTLs that match the CBM and VBM energy levels is critical to substantially reduce $V_{\text {oc }}$ loss in WB MHPs. In addition, the energy disorder of the ETL and HTL also affects the $V_{\mathrm{oc}}$. As an example, the benefit of the higher energy of the lowest unoccupied molecular orbital (LUMO) of indene- $\mathrm{C}_{60}$ 


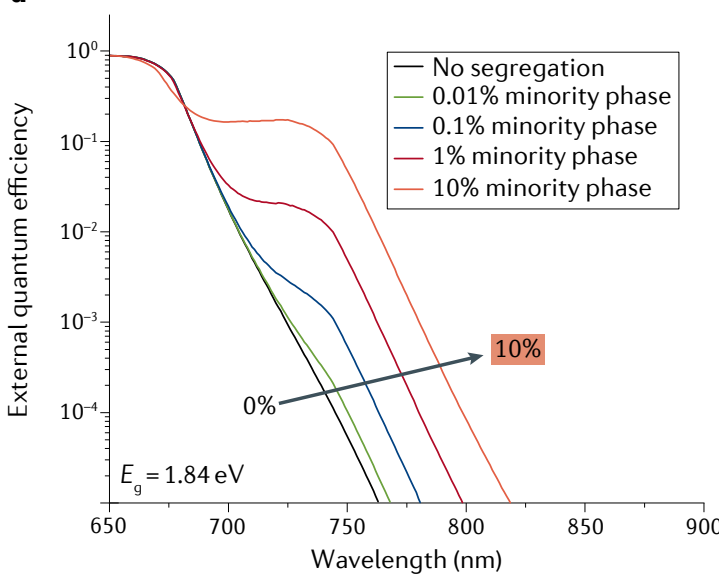

b

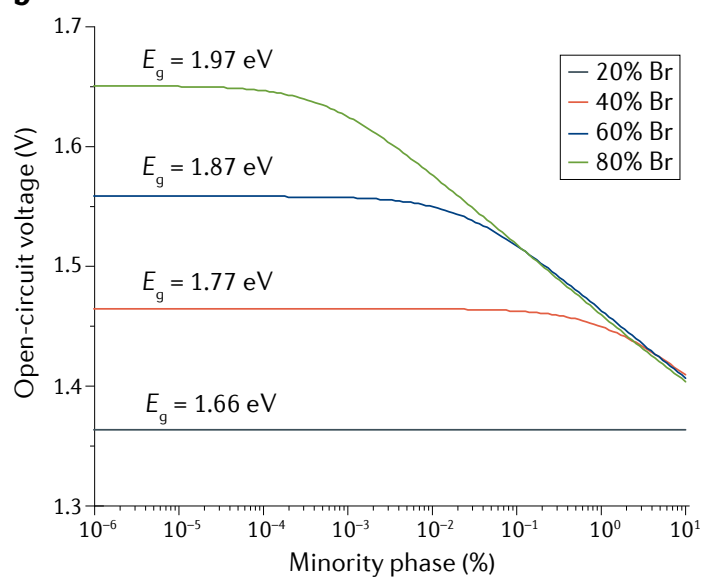

d

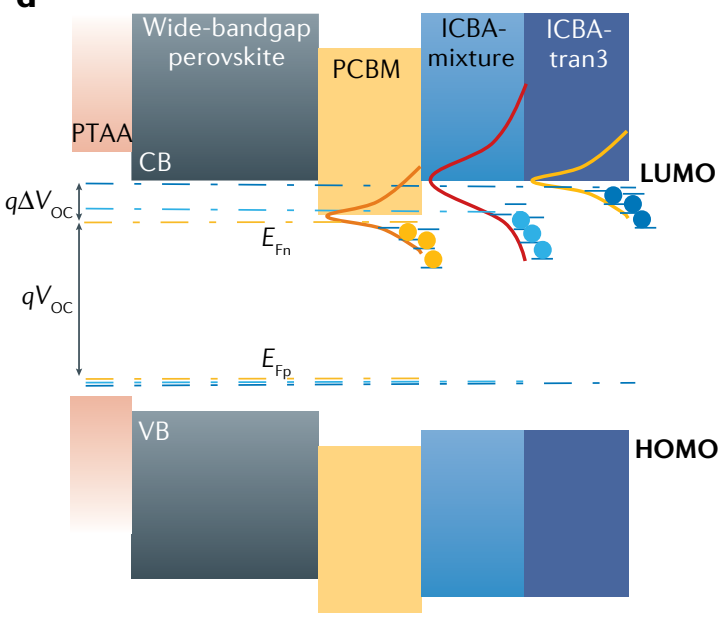

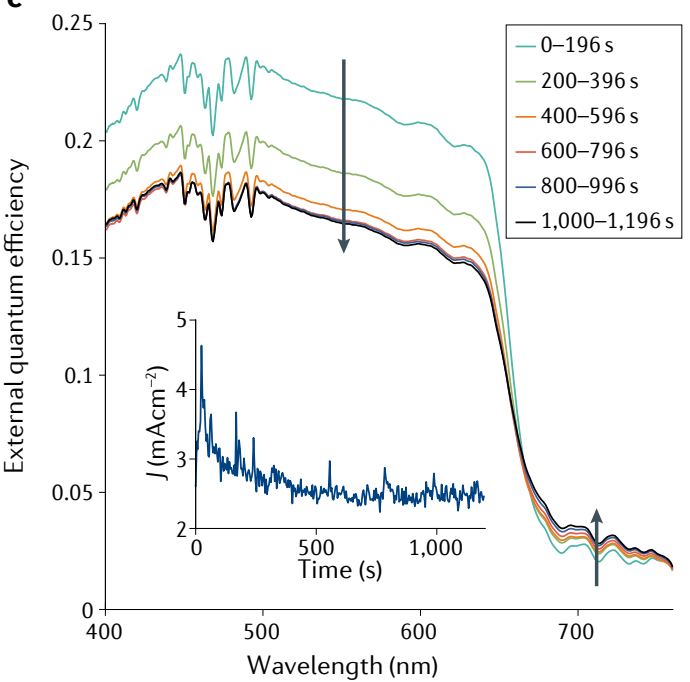

Fig. 5 | Impact of halide segregation and energy-level alignment on energy loss of wide-bandgap metal halide perovskite solar cells. a | Modelled external quantum efficiency (EQE) for perovskite solar cells with a bandgap $E_{g}$ of $1.84 \mathrm{eV}$ for a percentage of formed I-rich minority phase varying from $0 \%$ to $10 \%$. $\mathbf{b}$ | Radiative limit of the open-circuit voltage, $V_{o c}$, as a function of the volume percentage of minority phase (with an I-to-Br ratio of 4:1 in the minority phase). c| Evolution of the EQE spectra as a function of time from a mixed halide perovskite device under AM1.5 illumination at 0.6 Sun $\left(60 \mathrm{~mW} \mathrm{~cm}^{-2}\right)$ for a total time of $20 \mathrm{~min}$. The extracted current density J is plotted in the inset. $\mathbf{d} \mid$ Schematic illustration of how the energy level and energy disorder of electron transport layers influence the device $V_{\text {oc }}$ : a high energy level and a narrowly distributed density of states raise the quasi-Fermi level of the photogenerated electrons $\left(E_{F_{n}}\right)$ and thus increase the device $V_{\mathrm{o}} \cdot E_{\mathrm{Fp}}$ is the quasi-Fermi level of the photogenerated holes. Dashed lines represent the quasi-Fermi level of devices with [6,6]-phenyl- $\mathrm{C}_{61}$-butyric acid methyl ester (PCBM), with an indene- $\mathrm{C}_{60}$ bisadduct (ICBA) mixture, and with the ICBA-tran 3 isomer. CB, conduction band; HOMO, highest occupied molecular orbital; LUMO, lowest unoccupied molecular orbital; PTAA, poly[bis(4-phenyl)(2,4,6-trimethylphenyl)amine]; VB, valence band. Panels a and $\mathbf{b}$ reprinted with permission from REF. ${ }^{85}$, RSC. Panel $\mathbf{c}$ reprinted with permission from REF. ${ }^{64}$, Wiley. Panel $\mathbf{d}$ adapted with permission from REF. ${ }^{88}$, Wiley.

bisadduct (ICBA) is manifested by a larger $V_{\text {oc }}$ only after its energy disorder is minimized to be comparable to that of other fullerenes by isolating a specific isomer from the ICBA mixture when matching it with the WB MHP FA ${ }_{0.83} \mathrm{Cs}_{0.17} \mathrm{~Pb}\left(\mathrm{I}_{0.6} \mathrm{Br}_{0.4}\right)_{3}\left(\mathrm{REF}^{88}\right)$ (FIG. $5 \mathrm{~d}$ ).

\section{Overcoming halide segregation}

The stabilization of WB perovskites has made important steps forward, accompanied by an improved understanding of the defect photochemistry in this class of materials. Below we report a few approaches that target the reduction of native defects and/or the hampering of ion migration through lattice manipulation and passivation strategies. These approaches provide interesting guidelines for further developments, although it is difficult at this stage to pick a winning strategy: for this, a rationalization and quantification of the phenomena will be needed.

Lattice manipulation. The existence of polar A-site cations in MHPs aids halide migration by interacting dynamically with $\mathrm{V}_{\mathrm{I}}^{+}\left(\mathrm{REF}^{50}\right)$ and/or enhancing the electron-phonon coupling ${ }^{26}$. The photostability can be improved in thin films by using less-polar cations 
such as $\mathrm{FA}^{+}$and $\mathrm{Cs}^{+}$in the A-site ${ }^{23}$. Additionally, high external pressure helps to suppress halide segregation in $\mathrm{MAPb}\left(\mathrm{I}_{1-x} \mathrm{Br}_{x}\right)_{3}$, and inserting the smaller cation $\mathrm{Cs}^{+}$ has the same effect as imposing chemical compression on the lattice ${ }^{89}$. The much higher dipole moment of $\mathrm{MA}^{+}$ compared with $\mathrm{FA}^{+}$and $\mathrm{Cs}^{+}$was suggested to lead to its reorientation in response to the change of local electrical field due to adjacent deep trap formation, which could shift the deep trap states to shallower levels. Besides, the reorientation of MA dipoles can decrease the trap capture cross-section by screening carriers ${ }^{90}$. Therefore, a small amount of $\mathrm{MA}^{+}$can help to heal defects that introduce deep trap states without quantitatively affecting photostability. In line with these observations, almost all the recently reported tandem solar cells use mixed halide perovskites based on either Cs-FA or Cs-MA-FA as top absorbers, with an FA content generally above $65 \%$ and the rest being Cs and/or MA in the A-site ${ }^{3-18}$.

Fully inorganic lead halide perovskites based on Cs on the A-site $\left(\mathrm{CsPbI}_{x} \mathrm{Br}_{3-x}\right)$ also have potential as top absorbers in tandem solar cells, owing to their suitable bandgap and good thermal stability. Furthermore, $\mathrm{CsPbI} \mathrm{Br}_{3-\mathrm{x}}$ shows suppressed photoinduced halide segregation ${ }^{24}$. However, $\mathrm{CsPbI}_{x} \mathrm{Br}_{3-x}$ perovskites tend to have low phase stability, as the photoinactive yellow phase is thermodynamically favoured at room temperature, and the transition to the photoactive black phase needs high annealing temperature ${ }^{91-93}$. The progress of inorganic perovskite solar cells has been summarized in a few reviews ${ }^{94-96}$. Generally, the phase stability and photovoltaic performance of $\mathrm{CsPb}_{x} \mathrm{Br}_{3-x}$ perovskites still need to be improved to meet the requirements for top absorbers in tandem solar cells.

Another state-of-the-art strategy for minimizing the impact of halide segregation is to limit the $\mathrm{Br}$ ratio to $20 \%$ in the commonly used top absorbers, Cs-MA-FA MHPs $^{3,5-9,11,15,18}$. However, normally this strategy results in a limited bandgap of around $1.63 \mathrm{eV}$, which may sacrifice the transparency of the top absorber. Taking the widely studied perovskite/Si as an example, the theoretically estimated bandgap to perfectly match $\mathrm{Si}$ is $1.74 \mathrm{eV}$ (REF ${ }^{97}$ ). This value should be corrected to around $1.68 \mathrm{eV}$ by considering the deviation of realistic absorption from the ideal bandgap variation induced by band edge and temperature under practical working conditions ${ }^{98}$. Using a $1.63-\mathrm{eV}$ top absorber still causes a mismatch of about $0.05 \mathrm{eV}$. Several studies have shown that this minor mismatch can be compensated by composition modification of the perovskite. Alloying a large cation such as dimethylammonium with FA and Cs at the A-site may widen the bandgap, producing a 1.7-eV bandgap material with only $20 \% \mathrm{Br}\left(\right.$ REF $^{18}{ }^{18}$ ). Besides A cation alloying, successful $\mathrm{I} / \mathrm{Br} / \mathrm{Cl}$ alloying at the $\mathrm{X}$-site was demonstrated, extending the bandgap of $\mathrm{FA}_{0.75} \mathrm{Cs}_{0.25} \mathrm{~Pb}\left(\mathrm{I}_{0.8} \mathrm{Br}_{0.15}\right)_{3}$ from $1.63 \mathrm{eV}$ to $1.67 \mathrm{eV}\left(\mathrm{REF}^{3}{ }^{3}\right)$. Nevertheless, the development of perovskite/perovskite two-junction tandem solar cells and perovskite/ perovskite/perovskite (or $\mathrm{Si}$ ) three-junction tandem solar cells requires an optimal top absorber to have a bandgap of up to $1.8 \mathrm{eV}$ and $1.9 \mathrm{eV}\left(\mathrm{REF}^{97}\right)$, respectively; thus, in these systems, a higher $\mathrm{Br}$ fraction is necessary to promote the bandgap.
Morphology modification. Grain boundaries play a critical role in halide segregation in WB MHPs. Manipulating the grain size helps to suppress halide segregation. Since the initial report of the fabrication of large-grain WB MHP thin films by using a hydrophobic substrate ${ }^{99}$, multiple methods have been developed, including solvent annealing ${ }^{100}$ and the use of additives such as $\mathrm{Pb}(\mathrm{SCN})_{2}$ $\left(\mathrm{REFS}^{5,8,100,101}\right), \mathrm{MACl}$ and urea ${ }^{9}$. Non-radiative recombination is also effectively reduced and contributes to the lower $V_{\text {oc }}$ loss.

Owing to the volatility of organic cations, the grain surface tends to be $\mathrm{PbI}_{2}$-terminated, which may affect halide migration or carrier recombination for the following reasons: lower barrier for halide migration ${ }^{58}$; high density of traps that can induce local electric field by photocarrier trapping and drive halide migration ${ }^{42}$; and formation of $\mathrm{I}_{2}$ and metallic lead, which is detrimental to $V_{\text {oc }}$ and stability ${ }^{48,80,81}$. In MHPs, the surface termination can simply be manipulated by adjusting the $\mathrm{PbX}_{2}$-to- $\mathrm{AX}$ ratio through single-step deposition, as the self-assembly process tends to expel over-stoichiometric components into the grain boundaries ${ }^{102,103}$. In the case of sequential deposition, the residual $\mathrm{PbX}_{2}$ is possibly located at the bottom surface and the residual AX on the top surface ${ }^{104,105}$, whereas by increasing the annealing time or temperature the top surface and grain boundaries can be converted to the $\mathrm{PbX}_{2}$ termination ${ }^{73}$. $\mathrm{PbI}_{2}$ deficiency may make it trickier than $\mathrm{PbI}_{2}$ excess to achieve high efficiency; indeed, almost all demonstrated high efficiencies are for $\mathrm{PbI}_{2}$-excess systems in MHPs with bandgaps around $1.6 \mathrm{eV}$. One possible issue in the $\mathrm{PbI}_{2}$-deficient case is that small crystals stacking along the vertical direction can lead to poor extraction of photocarriers due to the hindering of the organic halide layer. An investigation of the combined effect of enlarging grain size and introducing excess FAX on the performance of solar cells based on WB MHPs ${ }^{101}$ demonstrated substantial reduction of $V_{\text {oc }}$ loss without sacrificing photocarrier extraction. Finally, additives such as KI (REF. $\left.{ }^{106}\right), \mathrm{MAH}_{2} \mathrm{PO}_{2}\left(\right.$ REF. $\left.^{9}\right)$, butylammonium halides ${ }^{107,108}$, benzylamine ${ }^{109,110}$ and phenethylammonium (PEA) salts $^{5,8}$, among others, have been successfully used to passivate surfaces and grain boundaries.

Ultimate stability and performance are expected to be reached with the development of monocrystalline WB MHPs. Much higher photostability has been demonstrated in a millimetre-sized single crystal of $\mathrm{MAPbI}_{2.1} \mathrm{Br}_{0.9}$ compared with a polycrystalline thin film ${ }^{53}$. Besides, the monocrystalline MHP showed a trap density three to four orders of magnitude lower, both in the bulk and at interfaces, compared with polycrystalline thin films ${ }^{71}$. However, spatially and temporally resolved fluorescence imaging shows that halide segregation still emerges in WB MHP monocrystalline microplates from crystal surfaces and edges ${ }^{51,52}$, which indicates that further surface passivation is necessary. Of course, the passivation should be much easier for monocrystalline than for polycrystalline MHPs, owing to the removal of embedded grain boundaries ${ }^{71}$. Generally, the fabrication of monocrystalline MHPs of sufficient quality for photovoltaics and the optimization of their performance and stability are still at a preliminary stage $\mathrm{e}^{111,112}$. 
Table 1 | Defect chemistry in Sn-based MHPs

$\begin{array}{ll}\text { p-doping } & \text { n-doping } \\ \mathrm{V}_{\mathrm{Sn}}^{0} \rightarrow \mathrm{V}_{\mathrm{Sn}}^{2-}+2 \mathrm{~h}^{+}(\mathrm{p} 1) & \mathrm{Sn}_{\text {bulk }}^{0} \rightarrow \mathrm{Sn}_{\mathrm{i}}^{2+}+2 \mathrm{e}^{-}(\mathrm{n} 1) \\ \mathrm{Sn}_{\mathrm{Sn}}^{2+} \rightarrow \mathrm{Sn}_{\mathrm{Sn}}^{0}+2 \mathrm{~h}^{+}(\mathrm{p} 2) & 2 \mathrm{l}_{\mathrm{i}}^{0} \rightarrow 2 \mathrm{~V}_{\mathrm{i}}^{+}+2 \mathrm{e}^{-}+\mathrm{I}_{2}(\mathrm{n} 2)\end{array}$

$\mathrm{I}_{2} \rightarrow 2 \mathrm{I}_{\mathrm{i}}^{-}+2 \mathrm{~h}^{+}(\mathrm{p} 3)$

Proposed defect chemistry processes leading to $\mathrm{p}$-type and n-type doping in $\mathrm{Sn}$-based metal halide perovskites ${ }^{119}$.

The fabrication and optimization are expected to be even more challenging for mixed $\mathrm{I}-\mathrm{Br}$ than for pure iodide MHPs, owing to the extra requirement of homogeneous distribution of halides in centimetre-sized samples. However, this research field is worth intensive efforts, as it holds promise for the production of highly stable and efficient tandem photovoltaics for commercialization.

\section{Tin-based narrow-bandgap MHPs}

\section{$\mathrm{Sn}^{2+}$ oxidation and related phenomena}

Defect activity and spontaneous p-type doping. Pure Sn MHPs have attracted intense attention since the demonstration of their capability as solar energy harvesters with an ideal bandgap of around $1.3 \mathrm{eV}$. Unfortunately, this absorber behaves like a metal rather than like a semiconductor in terms of electrical conductivity, which is highly correlated with its heavy p-type doping due to $\mathrm{Sn}^{2+}$ oxidation $^{21,113}$. Sn-based MHPs have a much lower redox potential of $\mathrm{Sn}^{2+} / \mathrm{Sn}^{4+}(+0.15 \mathrm{~V})$ than that of $\mathrm{Pb}^{2+} /$ $\mathrm{Pb}^{4+}(+1.67 \mathrm{~V})$ in $\mathrm{Pb}$-based $\mathrm{MHPs}^{22}$. Therefore, $\mathrm{Sn}^{2+}$ is easily oxidized to $\mathrm{Sn}^{4+}$, which leads to heavy p-type doping in Sn-based MHPs, whereas Pb-based MHPs show nearly intrinsic features. $\mathrm{O}_{2}$ is the most common oxidation agent that can reach $\mathrm{Sn}^{2+}$, and oxidation seems to be inevitable, as it proceeds even with $\mathrm{O}_{2}$ levels as low as a few ppm $^{114}$. As shown in Eq. (5), the oxidation of $\mathrm{Sn}^{2+}$ to $\mathrm{Sn}^{4+}$ is accompanied by removal of $\mathrm{Sn}$ atoms from the $\mathrm{ASnI}_{3}$ lattice to form $\mathrm{SnO}_{2}$, leading to the formation of $\mathrm{V}_{\mathrm{Sn}}{ }^{0}$ sites and, eventually, to the decomposition to the vacancy-ordered $\mathrm{A}_{2} \mathrm{SnI}_{6}$ double perovskite structure $^{114}$.

$$
\begin{aligned}
\mathrm{Sn}_{\mathrm{Sn}}(\mathrm{II})+\mathrm{O}_{2} & \rightarrow \mathrm{V}_{\mathrm{Sn}}^{0}+\mathrm{Sn}(\mathrm{IV}) \mathrm{O}_{2}+2 \mathrm{~h}^{+} \\
& \rightarrow \mathrm{V}_{\mathrm{Sn}}^{2-}+4 \mathrm{~h}^{+}+\mathrm{Sn}(\mathrm{IV}) \mathrm{O}_{2}
\end{aligned}
$$

DFT calculations confirmed that, even in the absence of external oxidizing agents, Sn-based MHPs tend to grow intrinsically $\mathrm{p}$-doped, owing to the high stability of $\mathrm{Sn}$ vacancies and halide interstitials, introducing holes according to processes $\mathrm{p} 1$ and $\mathrm{p} 3$ in TABLE $1^{115,116}$. The incorporation of $\mathrm{Sn}^{4+}$, due to the oxidation of precursors in imperfectly controlled synthesis conditions, may also contribute to aggravating the p-doping of Sn-based MHPs (process p2 in TABLE 1). Donor defects, such as Sn interstitials and halide vacancies, show higher formation energies than acceptor defects and are less active in mitigating the electronic disorder of Sn-based MHPs. For example, processes $n 1$ and $n 2$ in TABLE 1 are thermodynamically disfavoured ${ }^{117,118}$. DFT calculations also demonstrated that these defects introduce deep ionization levels in the $\mathrm{MASnI}_{3}$ perovskite bandgap and may be responsible for increased non-radiative recombination ${ }^{116}$

Interestingly, the defect chemistry of Sn-based MHPs appears profoundly different from that of lead-based perovskites, in which self $\mathrm{p}$-doping phenomena are absent and a more intrinsic behaviour is observed. DFT calculations showed that the different redox potential of $\mathrm{Pb}$ and $\mathrm{Sn}$ strongly influences the electronic features of the relative perovskites by modulating defect activity ${ }^{116}$. The inherently lower ionization potential of Sn-based MHPs, for example the higher absolute energy of the valence band compared with $\mathrm{Pb}$-based perovskites, is one of the factors at the origin of the high stability of Sn vacancies and thus of the p-doping of the material. Alloying of the $\mathrm{Sn}$ site by $\mathrm{Pb}$ progressively stabilizes the valence band of the perovskite and increases the stability of donor defects, by giving a more intrinsic character to the perovskite. This trend is illustrated in FIG. 6a, where the defect formation energies of native defects in $\mathrm{MA}(\mathrm{Pb}, \mathrm{Sn}) \mathrm{I}_{3} \mathrm{MHPs}$ are reported. The metal also largely affects the nature of deep traps in these materials (FIG. 6b). As discussed above, iodine-related defects, $\mathrm{I}_{\mathrm{i}}$ and $\mathrm{V}_{\mathrm{Pb}}$, are the main source of charge trapping in $\mathrm{Pb}$ MHPs, whereas donor defects such as $\mathrm{V}_{\mathrm{I}}$ and $\mathrm{Pb}_{\mathrm{i}}$ show a shallower behaviour. The higher band-edge energies of Sn MHPs suppress deep iodine traps and promote the formation of electron traps associated to $\mathrm{Sn}_{\mathrm{i}}$ and $\mathrm{V}_{\mathrm{I}}$. Interestingly, mixed $\mathrm{Pb} / \mathrm{Sn} \mathrm{MHPs}$ show an intermediate behaviour and are potentially free of deep traps.

Overall, these results suggest that to limit the self p-doping of Sn perovskites, two main strategies should be adopted: limiting the exposure to external oxidizing agents by a careful control of the synthesis conditions and by encapsulation; and developing chemical strategies aimed at stabilizing the Sn sublattice in the material.

Carrier lifetime and diffusion length. The high background hole concentration in Sn-based MHPs leads to very short carrier lifetime and diffusion length, resulting in substantially deteriorated photovoltaic performance with respect to $\mathrm{Pb}$-based MHPs. The background hole concentration in Sn-based MHPs made of polycrystalline thin films can reach $10^{20} \mathrm{~cm}^{-3}$, leading to strong monomolecular charge-carrier decay from electron recombination ${ }^{119-122}$. Consequently, the carrier lifetime is tens to several hundreds of picoseconds, which is considerably shorter than that of Pb-based MHPs (typically 100-1,000 ns). Besides, the high lattice disorder due to $\mathrm{Sn}^{2+}$ oxidation leads to a low carrier mobility of several $\mathrm{cm}^{2} \mathrm{~V}^{-1} \mathrm{~s}^{-1}\left(\mathrm{REFS}^{119,120,123}\right)$. As a result, the carrier diffusion length is only tens of nanometres compared with over a micrometre in $\mathrm{Pb}$-based MHPs, which limits the carrier extraction in solar cells. Simulations of the extraction diffusion length in $\mathrm{CH}_{3} \mathrm{NH}_{3} \mathrm{SnI}_{3}$ as a function of the background concentration of doped holes suggest that on reducing the doping level in pure Sn MHPs to $\sim 10^{15} \mathrm{~cm}^{-3}$, the diffusion length may approach that of very efficient $\mathrm{Pb}$-based MHPs ${ }^{120}$. Such a doping reduction can be achieved, for example, by improving the crystalline quality and reducing grain boundaries. The use of ingots of pure Sn-based MHPs containing high-quality large 

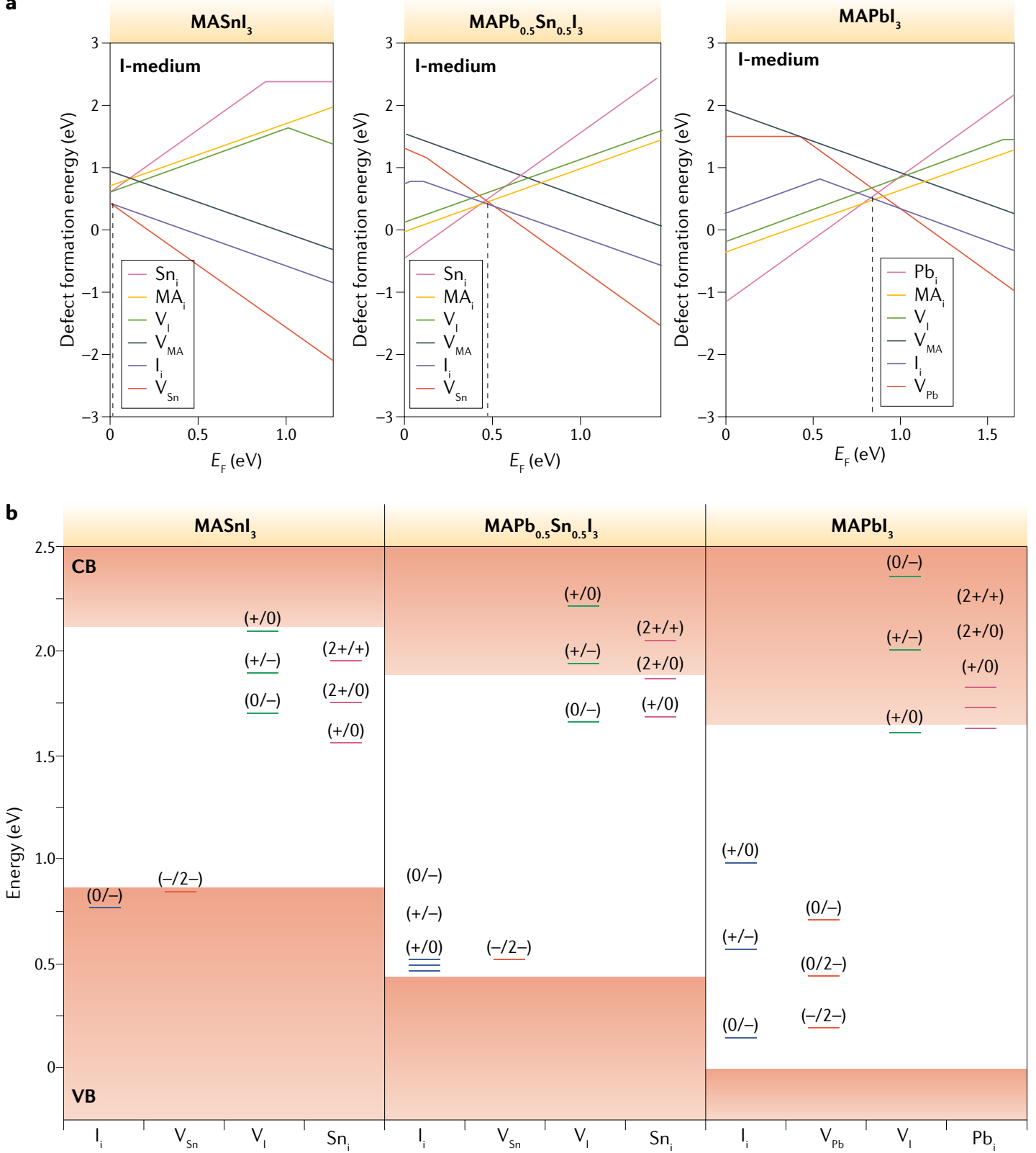

Fig. 6 Impact of alloying of the Sn site by Pb on point defects. a | Defect formation energies in MASnl $\mathrm{MAPb}_{0.5} \mathrm{Sn}_{0.5} \mathrm{I}_{3}$ and $\mathrm{MAPbl}_{3}$ perovskites in I-medium conditions of growth. $\mathbf{b}$ | Thermodynamic ionization levels of main deep traps in these systems. CB, conduction band; $E_{\mathrm{p}}$ Fermi energy; $\mathrm{I}_{\mathrm{i}}$, interstitial I; VB, valence band; $\mathrm{V}_{\mathrm{X}}, \mathrm{X}_{\mathrm{Vacancy}} \mathrm{X}_{\mathrm{i}}$, interstitial $\mathrm{X}$. Panels a and $\mathbf{b}$ adapted with permission from REF. ${ }^{116}$, ACS.

single crystals ${ }^{123}$, or of pure Sn MHP thin films prepared with purified $\mathrm{SnI}_{2}$ and $\mathrm{SnF}_{2}$ additives, does indeed reduce the background hole concentration to $10^{17} \mathrm{~cm}^{-3}$ (REF. ${ }^{124}$ ), extending the carrier diffusion length to $500-1,000 \mathrm{~nm}$. $\mathrm{GeI}_{2}$-doped Sn-based MHP thin films with reduced lattice disorder and a doping density of $10^{15} \mathrm{~cm}^{-3}$ were also obtained by incorporating an ethylammonium cation and an $\mathrm{SnF}_{2}$ additive ${ }^{125}$.

Large Stokes shift and high-level tail states. Sizable Stokes shifts of 200-250 meV are found in Sn-based MHPs between the absorption onset centre and the PL peak emission energy ${ }^{119,122}$, whereas for $\mathrm{MAPbI}_{3}$ Stokes shifts of at most $10 \mathrm{meV}$ have been reported ${ }^{126}$. The high energetic disorder in Sn-based MHPs due to $\mathrm{Sn}^{2+}$ oxidation possibly extends tail states into the bandgap, leading to a considerable redshift of the peak PL energy from the absorption onset, because carriers tend to thermalize into the tail states. This prominent Stokes shift can lead to high $V_{\text {oc }}$ loss, as the reverse dark saturation current increases substantially.

\section{Strategies to overcome $\mathrm{Sn}^{2+}$ oxidation}

Reducing additives. One of the established techniques to reduce $\mathrm{Sn}^{2+}$ oxidation points at the use of additives such as $\mathrm{SnX}_{2}(\mathrm{X}=\mathrm{F}, \mathrm{Cl} \text { or I })^{127-129}$. The introduction of extra $\mathrm{Sn}^{2+}$ limits the formation of $\mathrm{Sn}$ vacancies and increases the oxidation potential of $\mathrm{Sn}^{2+}$, leading to the suppressed 
formation of local structures of $\mathrm{A}_{2} \mathrm{SnI}_{6}$ due to $\mathrm{ASnI}_{3}$ oxidation. Therefore, the acceptor concentration of $\mathrm{Sn}^{4+}$ / $\mathrm{V}_{\mathrm{Sn}}{ }^{0}$ decreases, reducing the high background hole concentration. As a matter of fact, an optimized content of $20 \% \mathrm{SnF}_{2}$ additive can reduce the hole concentration by two orders of magnitude ${ }^{121}$. The carrier lifetime and electron mobility are much improved, extending the diffusion length to over $500 \mathrm{~nm}$. Besides, the Stokes shift is reduced considerably from $250 \mathrm{meV}$ in heavily doped $\mathrm{ASnI}_{3}$ to around $40 \mathrm{meV}$ by adding $10 \% \mathrm{SnF}_{2}$ (REF. ${ }^{124}$ ). As a result of the improved optoelectronic properties of the material, both the carrier extraction and $V_{\mathrm{oc}}$ are enhanced. However, the ratio of $\mathrm{SnF}_{2}$ additive is critical, because the formation of $S n_{i}, V_{I}$ and $S n_{I}$ antisites with energy levels deep in the bandgap may become prominent when the ratio exceeds some threshold. Excess $\mathrm{SnF}_{2}$ may also induce inhomogeneity in the film, reducing the performance of the resulting photovoltaic devices. Meanwhile, $\mathrm{SnX}_{2}$ additives easily undergo oxidation themselves when exposed to oxygen, which indicates their limited capability of removing oxidation in $\mathrm{ASnI}_{3}$ alone $^{130}$. Moreover, the monomolecular charge-carrier recombination does not approach zero but a value of $1.2 \times 10^{9} \mathrm{~s}^{-1}$ as the acceptor concentration tends to zero, equivalent to a maximum lifetime of the order of a nanosecond ${ }^{122}$. This observation suggests the existence of an additional trap-mediated charge recombination mechanism that is not remedied by $\mathrm{SnF}_{2}$, related perhaps to $\mathrm{Sn}$ interstitials and I vacancies, as suggested by DFT calculations ${ }^{115,116}$. Various reducing agents have been explored as co-additives to reduce the oxidation in pure Sn-based MHPs, including hydrazine vapour, pyrazine, piperazine and hydroxybenzene sulfonic acid ${ }^{129,131-133}$. Other effective approaches to reduce self-doping of the material by mitigating the formation of $\mathrm{Sn}$ vacancies include the addition of the metal ion $\mathrm{Cd}^{2+}\left(\mathrm{REF}^{16}{ }^{16}\right)$ and the use of metallic $\mathrm{Sn}$ powders within the precursor solution to reduce $\mathrm{Sn}^{4+}$ to $\mathrm{Sn}^{2+}$ via a comproportionation reaction. For example, the charge-carrier density of $\mathrm{FASnI}_{3}$ films is reduced by one order of magnitude when excess metallic $\mathrm{Sn}$ is used ${ }^{134}$.

A-site modification. The $\mathrm{Sn}^{2+} / \mathrm{Sn}^{4+}$ oxidation process is affected also by the type of cation at the A-site. A study of the formation energy of $\mathrm{Sn}$ vacancies in $\mathrm{MASnI}_{3}$ and $\mathrm{FASnI}_{3}$ by DFT calculations ${ }^{115}$ demonstrated that $\mathrm{FASnI}_{3}$ presents higher formation energy of Sn vacancies than $\mathrm{MAPbI}_{3}$, owing to the large size of $\mathrm{FA}^{+}$and weaker antibonding coupling between $\mathrm{Sn} 5 s$ and I $5 p$. The same phenomenon was observed with combined oxidation tests and DFT calculations, revealing that the change from MA to FA cations considerably modifies the electronic structure of the perovskite lattice and reduces the degree of oxidation ${ }^{114}$. Moreover, the carrier lifetime is increased by substituting MA with FA on the A-site, owing to improved film morphology and reduced charge-carrier recombination ${ }^{135}$. These observations explain why $\mathrm{FASnI}_{3}$-based solar cells generally perform better than $\mathrm{MASnI}_{3}$-based solar cells, showing a reported PCE of up to $12.4 \%$ compared with the $7.1 \%$ achieved in $\mathrm{MASnI}_{3}$-based solar cells ${ }^{22,136}$. A slightly larger guanidinium $\left(\mathrm{CH}_{6} \mathrm{~N}_{3}{ }^{+}, \mathrm{GA}\right)$ cation of approximate size $278 \mathrm{pm}$ (compared with about $253 \mathrm{pm}$ for $\mathrm{FA}^{+}$) can substitute up to $30 \%$ of $\mathrm{FA}^{+}$in $\mathrm{FASnI}_{3}$ without changing the lattice structure ${ }^{137}$. This substitution further reduces the antibonding coupling between $\mathrm{Sn} 5 s$ and I $5 p$, and thus reduces $S n$ vacancy formation, leading to highly improved PCE and stability. Interestingly, even larger cations such as ethylenediammonium can be accommodated in the Sn-based MHP lattice and maintain the 3D connection by expelling $\mathrm{Sn}$ and $\mathrm{X}$ atoms and forming some kind of 'hollow' structure ${ }^{138}$. These hollow perovskites possess surfaces terminated by the large organic cation, in a similar way to $2 \mathrm{D}$ perovskites, while maintaining good carrier transport like 3D perovskites. As a result, Sn-based MHPs with hollow structure exhibit extremely high air stability and decent efficiency ${ }^{139}$.

Morphology modification and passivation. Bulk crystals of $\mathrm{ASnI}_{3}$ exhibit a background hole concentration of the order of $10^{17} \mathrm{~cm}^{-3}\left(\mathrm{REFS}^{123,140}\right)$, which is generally two to three orders of magnitude lower than that of $\mathrm{ASnI}_{3}$ thin films ${ }^{121,122}$. This indicates that the surface and grain boundaries may play a critical role in $\mathrm{Sn}^{2+}$ oxidation. Considering the ideal case without oxidation, an organic tin halide perovskite may also show a $\mathrm{SnI}_{2}$-terminated surface, owing to the possible loss of volatile organic species. Once exposed to oxygen, the open surface is easily oxidized without the protection of organic cations, leading to the formation of Sn vacancies, which in turn possibly provide the pathway for oxygen invasion towards the bulk, accelerating the oxidation. In addition, the oxidation of the open surface introduces deep traps and substantially offsets the energy levels, leading to poor carrier extraction and collection. Therefore, fabrication of compact thin films with reduced grain boundaries and well-passivated open surfaces is extremely important for the minimization of oxidation. However, it is more challenging to achieve good film morphology for Sn-based MHPs than for their Pb-based counterparts, owing to the fast reaction between $\mathrm{SnI}_{2}$ and MAI/FAI. Many attempts have been made to slow the reaction between $\mathrm{SnI}_{2}$ and MAI/FAI and obtain highquality films: methods include solvent engineering, hot anti-solvent casting, cation exchange and vapour deposition $^{139,141}$. Various additives or additive combinations have also been explored to further promote improved morphology and/or surface passivation ${ }^{142}$. The formation of $2 \mathrm{D} / 3 \mathrm{D}$ structures, the addition of large cations such as $\mathrm{PEA}^{+}$, butylammonium $\left(\mathrm{BA}^{+}\right)$and ammonium valeric acid $\left(\mathrm{AVA}^{+}\right)$into the precursor solution, and the introduction of large cations on the surface by post-surface treatment with PEABr or ethylenediamine also help to reduce oxidation and the generation of deep traps, as these large cations tend to align compactly on the surface terminations ${ }^{139,141}$.

Energy-level alignment. It is more challenging to realize good energy-level alignment at the interface with transporting layers for pure Sn MHPs than for Pb-based materials. The surface energy level of pure Sn MHPs can be offset substantially owing to oxidation. Severe oxidation of $\mathrm{Sn}^{2+}$ was reported to shift the valence band from $-4.88 \mathrm{eV}$ to $-6.0 \mathrm{eV}\left(\right.$ REF $\left.^{135}\right)$. Furthermore, Sn-based 


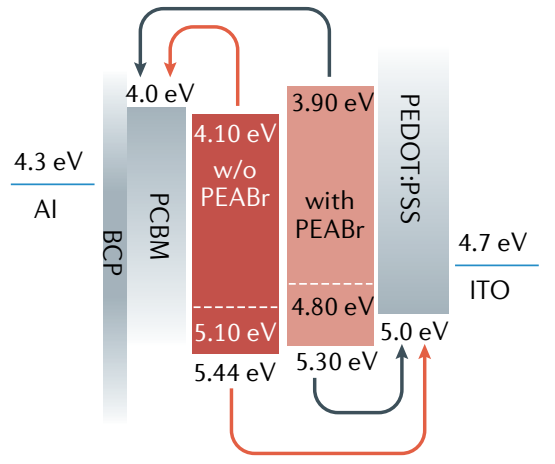

b

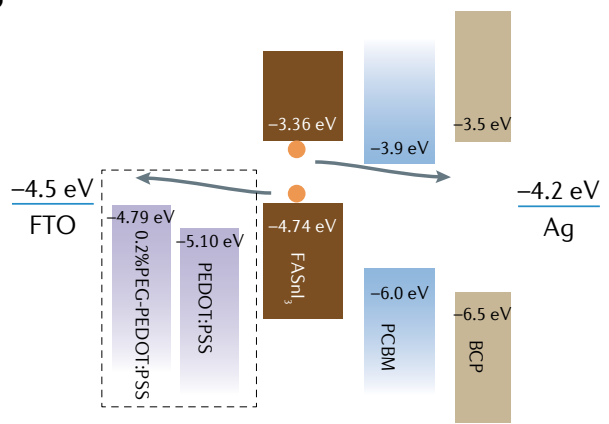

C

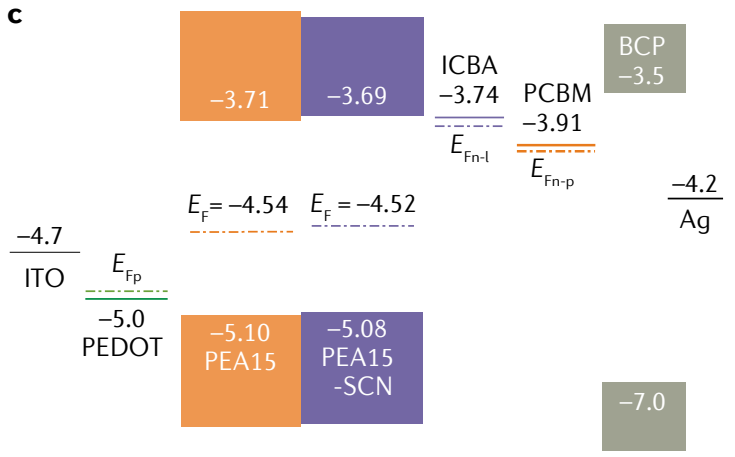

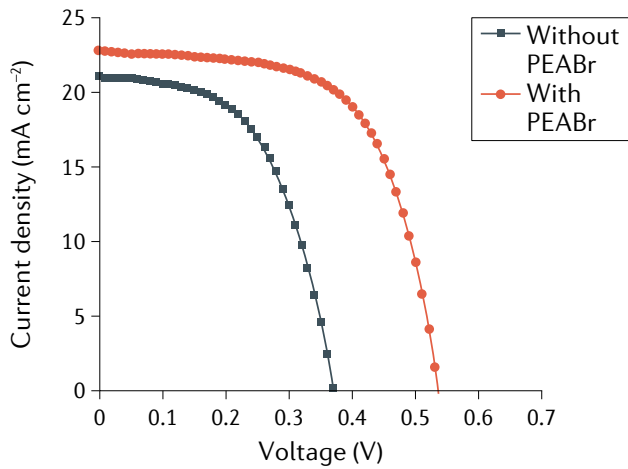

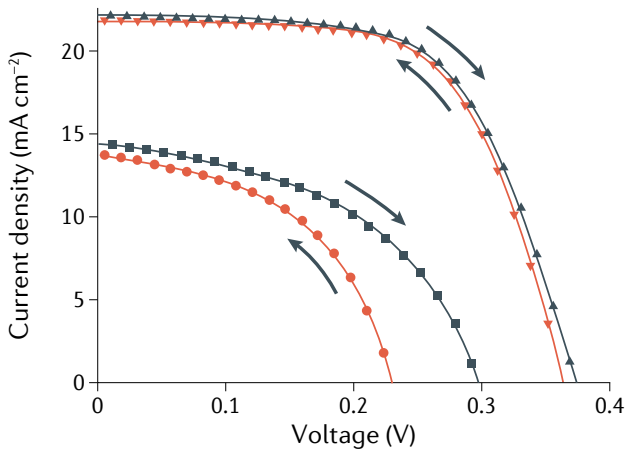

$\rightarrow-$ PEDOT:PSS forward $\rightarrow-$ PEDOT:PSS reverse $\leftarrow 0.2 \%$ PEDOT:PSS forward $\rightarrow 0.2 \%$ PEDOT:PSS reverse

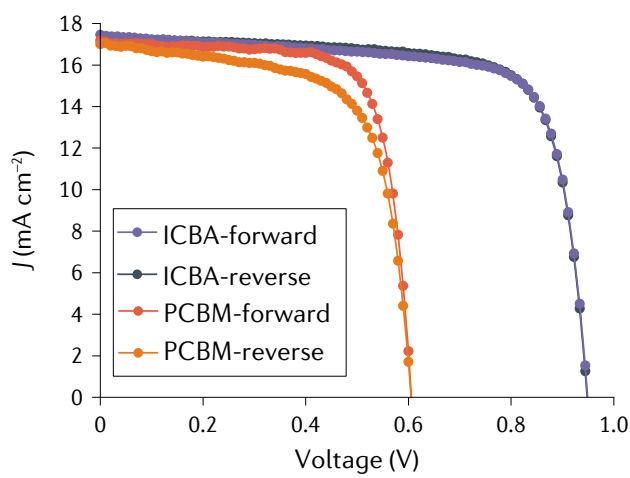

Fig. 7 | Interface modulation in FASnl ${ }_{3}$ MHP solar cells. a | Energy-level diagram (left) and current-density-voltage (U-V) curves (right) of $\mathrm{FASnl}_{3}$ inverted metal halide perovskite (MHP) solar cells without and with PEABr treatment. b | Energy diagram (left) and $J-V$ curves (right) of $\mathrm{FASnl}_{3}$ MHP solar cells based on poly(3,4-ethylenedioxythiophene) polystyrene sulfonate (PEDOT:PSS) and polyethylene glycol (PEG)-PEDOT:PSS. c | Schematic illustration of energy levels (left) and J-V curves (right) of certified MHP solar cells with indene- $\mathrm{C}_{60}$ bisadduct (ICBA) and [6,6]-phenyl- $\mathrm{C}_{61}$-butyric acid methyl ester (PCBM). The perovskite absorber material is $\mathrm{PEA}_{015} \mathrm{FA}_{0.85} \mathrm{Snl}_{3}$, and $\mathrm{NH}_{4} \mathrm{SCN}$ is used as additive. $\mathrm{BCP}, 2,9$-dimethyl4,7-diphenyl-1,10-phenanthroline; $E_{F}$, Fermi energy; FTO, fluorine-doped tin oxide; ITO, indium tin oxide; PEA, phenethylammonium; . Panel $\mathbf{a}$ adapted with permission from REF. ${ }^{143}$, Wiley. Panel $\mathbf{b}$ adapted with permission from REF. ${ }^{145}$, ACS. Panel c reprinted from REF.22, CC BY 4.0 (https://creativecommons.org/licenses/by/4.0/).

perovskites have shallower conduction and valence bands than $\mathrm{Pb}$-based perovskites. The estimated conduction and valence bands of $\mathrm{ASnI}_{3}\left(\mathrm{~A}=\mathrm{MA}^{+}, \mathrm{FA}^{+}\right.$and $\left.\mathrm{Cs}^{+}\right)$ are located at -3.49 to $-3.28 \mathrm{eV}$ and -4.75 to $-4.58 \mathrm{eV}$, respectively ${ }^{139}$. As a result, a large gap of over $0.7 \mathrm{eV}$ is created between the conduction band of $\mathrm{ASnI}_{3}$ and that of commonly used ETLs like $\mathrm{TiO}_{2}$ and $\mathrm{SnO}_{2}$, which largely contributes to the band alignment mismatch. In addition, the valence band of HTLs is typically deeper than that of $\mathrm{ASnI}_{3}$, blocking hole transfer and leading to inefficient photocarrier extraction and thus $J_{\mathrm{sc}}$ and $V_{\text {oc }}$ losses. Therefore, to improve the performance of Sn-based perovskite solar cells, the modification of the surface energy level of $\mathrm{ASnI}_{3}$ and the selection of ETLs and HTLs with suitable LUMO and HOMO (highest occupied molecular orbital) are very important. 
Surface modification by PEABr improves the band alignment at both the $\mathrm{FASnI}_{3} / \mathrm{PCBM}^{143}$ (FIG. 7a) and PEDOT:PSS/FASnI ${ }_{3}\left(\right.$ REF. $^{144}$ ) interfaces (where PCBM is $[6,6]$-phenyl- $\mathrm{C}_{61}$-butyric acid methyl ester and PEDOT:PSS is poly(3,4-ethylenedioxythiophene) polystyrene sulfonate). In addition, the discrepancy between the HOMO of PEDOT:PSS and the valence band of $\mathrm{FASnI}_{3}$ can be reduced by introducing $0.2 \%$ polyethylene glycol (PEG) into PEDOT:PSS, considerably enhancing the $J-V$ performance (FIG. $7 \mathrm{~b}$ ) and reducing the large hysteresis caused by misaligned energy levels ${ }^{145}$. Transport materials with suitable HOMO, such as triphenylamine ${ }^{146}$, and suitable LUMO, such as $\mathrm{ZnS}$ $\left(\right.$ REF $\left.^{147}\right)$ or ICBA ${ }^{127}$, have been explored to match with $\mathrm{ASnI}_{3}$. Recently, Zhijun Ning and co-workers replaced PCBM with ICBA to match $\mathrm{FASnI}_{3}$ for electron extraction (FIG. 7c), achieving a substantial improvement of $V_{\text {oc }}$ from $0.6 \mathrm{~V}$ to $0.94 \mathrm{~V}$ and a PCE of $12.4 \%$, higher than previous values for Sn-based solar cells ${ }^{22}$. The authors clarified that the lower Fermi level (by $80 \mathrm{meV}$ ) and shallower LUMO level of ICBA compared with PCBM possibly lead to lower electron density, which can reduce interface carrier recombination with p-type $S n$ perovskite films, contributing to the photovoltaic performance improvement. This is about 30\% higher than the PCE of the highest-efficiency Sn perovskite solar cells previously reported, demonstrating that the deep LUMO of the ETL and the energy-band misalignment are important factors limiting the $V_{\mathrm{oc}}$.

\section{Mixed $\mathrm{Sn}-\mathrm{Pb}$ perovskites}

The progress of mixed $\mathrm{Sn}-\mathrm{Pb}$ perovskites $\left(\mathrm{ASn}_{x} \mathrm{~Pb}_{1-x} \mathrm{I}_{3}\right)$ benefited from the development of pure $\mathrm{Sn}$ perovskites. The bandgap shows a bowing trend with decreasing $\mathrm{Sn}$-to- $\mathrm{Pb}$ ratio in the perovskite lattice, narrowing from $1.3-1.4 \mathrm{eV}$ down to a minimum of about $1.2 \mathrm{eV}$ as $x$ decreases from 1 to 0.6 (REF. ${ }^{148}$ ). This makes the $\mathrm{ASn}_{x} \mathrm{~Pb}_{1-x} \mathrm{I}_{3}$ perovskite with $x \approx 0.6$ a good absorber for tandem solar cells, because it can provide a high PCE limit when combined with a bandgap-matched top absorber. A decrease in the bandgap of the WB top absorber needs to happen in concert with a decrease in the bandgap of the bottom absorber, which enables the use of WB perovskites with lower Br ratio and thus lower energy loss and higher stability. Furthermore, the oxidation of $\mathrm{Sn}^{2+}$ is substantially reduced when part of the $\mathrm{Sn}$ is replaced with $\mathrm{Pb}\left(\mathrm{REF}^{149}\right)$. $\mathrm{Sn}-\mathrm{Pb}$ binary perovskites oxidize more slowly than Sn perovskites not simply because there is less Sn to oxidize, but also because the degradation mechanism is fundamentally different, as the valence band is substantially downshifted by alloying $\mathrm{Sn}$ with $\mathrm{Pb}$, shifting the oxidation potential of the perovskite towards that of the pure $\mathrm{Pb}$-based material, which is more resilient to oxidation than the Sn-based one. Besides, the deposition of mixed $\mathrm{Sn}-\mathrm{Pb}$ perovskites is easier to handle and leads to much better morphology than pure Sn perovskites. Consequently, the carrier lifetime and diffusion length reach values of over $1 \mu \mathrm{s}$ and $3 \mu \mathrm{m}$, respectively, which are much higher than the highest reported values for pure Sn perovskites ${ }^{14,15}$.

An efficiency of $21.7 \%$ has been reported for a single-junction $\mathrm{FA}_{0.7} \mathrm{MA}_{0.3} \mathrm{~Pb}_{0.5} \mathrm{Sn}_{0.5} \mathrm{I}_{3}$ perovskite solar cell $^{12}$, which is far beyond the values of up to $12.4 \%^{22}$ reported for pure $\mathrm{Sn}$ perovskite solar cells. Mixed $\mathrm{Sn}-\mathrm{Pb}$ perovskites also have potential for efficient all-perovskite tandem solar cells, reaching a certified efficiency of $24.8 \%{ }^{14}$. Despite the extremely fast progress, Sn-based materials suffer from poor reproducibility owing to the high tendency of $\mathrm{Sn}^{2+}$ to oxidize, so that even a trace amount of $\mathrm{O}_{2}$ during fabrication can strongly affect the final device performance. In addition, most of the high-performance mixed $\mathrm{Sn}-\mathrm{Pb}$ perovskites reported so far contain the volatile $\mathrm{MA}^{+}$cation and the organic PEDOT:PSS as hole transport material, causing concerns in terms of thermal stability of the NB MHP device as a whole. MA-free and PEDOT:PSS-free mixed $\mathrm{Sn}-\mathrm{Pb}$ solar cells that passed $1,000 \mathrm{~h}$ thermal and light stability tests were recently demonstrated, suggesting a promising path to achieve thermally stable devices ${ }^{150,151}$. However, the maximum PCE achieved with such MHP composition is still lower than that of the archetypal $\mathrm{Pb}$-based perovskites, owing to a large $V_{\text {oc }}$ deficit and low fill factors. More effort should be made to fundamentally understand the mechanism associated with instability and carrier transport dynamics in mixed $\mathrm{Sn}-\mathrm{Pb}$ MHPs to improve the perovskite bulk properties, reduce dark saturation current densities in regions near grain boundaries and push the PCE of all-perovskite tandem solar cells to $30 \%$, as well as fostering reproducibility in the field.

\section{Future perspectives}

The chemical composition of halide perovskites can be widely tuned to form materials with very different energy bandgaps, spanning from the near infrared to the ultraviolet. This versatility is a great advantage for fully exploiting the exceptional optoelectronic properties of halide perovskite materials. There are, however, many crucial aspects that need to be addressed, above all the material instability under operational conditions. In WB perovskites, the presence of defects and their migration make the material unstable, especially under light soaking. Grain boundaries and the surface act as defect reservoirs by providing stabilizing sites for defect nucleation that initiate the halide segregation process. Therefore, the most common and effective approaches to stabilize the bandgap look at either reducing the native defects or hampering the ion migration through lattice manipulation and passivation strategies. Despite the great improvements in this regard, it is worth noting that the large $V_{\mathrm{oc}}$ deficit of WB halide perovskites calls for an investigation of losses, which probably are not represented only by simple halide segregation.

Sn-based halide perovskites have a much lower ionization potential than $\mathrm{Pb}$-based materials, causing high stability of Sn vacancy defects and thus promoting the formation of electron traps within the bandgap. This intrinsic material property, which is related to the fundamentally different energy of $\mathrm{Sn} 5 s$ and $\mathrm{Pb} 6 s$ orbitals (the reason that we use $\mathrm{Pb}$ and not $\mathrm{Sn}$ for car batteries), makes stabilizing Sn-based perovskites highly challenging. When $\mathrm{Sn}$ is partly replaced by $\mathrm{Pb}$, an interesting compound is formed, which calls for deeper investigations of 
its electronic and physical chemical properties. Indeed, PCEs as high as $21.1 \%$ have been achieved with $50 \% \mathrm{~Pb}$ content. The research community has made great efforts to limit the oxidation process of $\mathrm{Sn}^{2+}$ and formation of $\mathrm{Sn}^{4+}$, mainly through the use of additives and reducing agents. Still, the chemistry of the oxidation process and its impact on carrier dynamics and recombination is poorly understood, if at all addressed. So far, the main barrier is a poor understanding of the chemistry of these compounds, the role of additives, and the stability and reproducibility of the samples.
Overall, MHPs are still far from being defect-tolerant, where tolerance is defined not only in terms of efficiency but also stability. Impressive work has been conducted so far on $\mathrm{MAPbI}_{3}$ and similar compounds with bandgap fixed around $1.6 \mathrm{eV}$. On these materials we have built most of our knowledge and achieved solid success. Now it is crucial to extend this knowledge to a broader library of materials to exploit one of the main properties of halide perovskites: versatility.

Published online: 22 June 2021
1. Kojima, A., Teshima, K., Shirai, Y. \& Miyasaka, T. Organometal halide perovskites as visible-light sensitizers for photovoltaic cells. J. Am. Chem. Soc. 131, 6050-6051 (2009).

2. Shockley, W. \& Queisser, H. J. Detailed balance limit of efficiency of $\mathrm{p}-\mathrm{n}$ junction solar cells. J. Appl. Phys. 32, 510-519 (1961)

3. $\mathrm{Xu}, \mathrm{J}$. et al. Triple-halide wide-band gap perovskites with suppressed phase segregation for efficient tandems. Science 367, 1097-1104 (2020).

4. Chen, B. et al. Blade-coated perovskites on textured silicon for $26 \%$-efficient monolithic perovskite/silicon tandem solar cells. Joule 4, 850-864 (2020).

5. Kim, D. et al. Efficient, stable silicon tandem cells enabled by anion-engineered wide-bandgap perovskites. Science 368, 155-160 (2020).

6. Chen, B. et al. Enhanced optical path and electron diffusion length enable high-efficiency perovskite tandems. Nat. Commun. 11, 1257 (2020).

7. Hou, Y. et al. Efficient tandem solar cells with solutionprocessed perovskite on textured crystalline silicon. Science 367, 1135 (2020)

8. Kim, D. H. et al. Bimolecular additives improve wideband-gap perovskites for efficient tandem solar cells with CICS. Joule 3, 1734-1745 (2019).

9. Chen, B. et al. Grain engineering for perovskite/silicon monolithic tandem solar cells with efficiency of $25.4 \%$ Joule 3, 177-190 (2019)

10. Li, Z et al. Wide-bandgap perovskite/gallium arsenide tandem solar cells. Adv. Energy Mater. 10, 1903085 (2019).

11. Abdollahi, N. B. et al. Vacuum-assisted growth of low-bandgap thin films ( $\mathrm{FA}_{0} \mathrm{MA}_{02} \mathrm{Sn}_{05} \mathrm{~Pb}_{0} \mathrm{I}_{3}$ ) for all-perovskite tandem solar cells. Adv. Energy Mater 10, 1903583 (2020)

12. Xiao, K. et al. All-perovskite tandem solar cells with $24.2 \%$ certified efficiency and area over $1 \mathrm{~cm}^{2}$ using surface-anchoring zwitterionic antioxidant. Nat. Energy 5, 870-880 (2020)

13. Yu, Z. et al. Simplified interconnection structure based on $\mathrm{C}_{60} / \mathrm{SnO}_{2-x}$ for all-perovskite tandem solar cells. Nat. Energy 5, 657-665 (2020)

14. Lin, R. et al. Monolithic all-perovskite tandem solar cells with $24.8 \%$ efficiency exploiting comproportionation to suppress $\mathrm{Sn}$ (ii) oxidation in precursor ink. Nat. Energy 4, 864-873 (2019).

15. Tong, J. et al. Carrier lifetimes of $>1 \mu \mathrm{s}$ in $\mathrm{Sn}-\mathrm{Pb}$ perovskites enable efficient all-perovskite tandem solar cells. Science 364, 475-479 (2019).

16. Yang, Z. et al. Enhancing electron diffusion length in narrow-bandgap perovskites for efficient monolithic perovskite tandem solar cells. Nat. Commun. 10, 4498 (2019).

17. McMeekin, D. P. et al. Solution-processed all-perovskite multi-junction solar cells. Joule 3 387-401 (2019)

18. Palmstrom, A. F. et al. Enabling flexible all-perovskite tandem solar cells. Joule 3, 2193-2204 (2019).

19. Jiang, Q. et al. Surface passivation of perovskite film for efficient solar cells. Nat. Photonics 13, 460-466 (2019).

20. Hoke, E. T. et al. Reversible photo-induced trap formation in mixed-halide hybrid perovskites for photovoltaics. Chem. Sci. 6, 613-617 (2015).

21. Takahashi, Y. et al. Charge-transport in tin-iodide perovskite $\mathrm{CH}_{3} \mathrm{NH}_{3} \mathrm{Snl}_{3}$ : origin of high conductivity. Dalton T. 40, 5563-5568 (2011).

22. Jiang, X. et al. Ultra-high open-circuit voltage of tin perovskite solar cells via an electron transporting layer design. Nat. Commun. 11, 1245 (2020).

This work clarifies the mechanism for the low voltage of tin perovskite solar cells and presents good energy-level alignment at the interface between the pure tin perovskite and the electron transporting layer, achieving high open-circuit voltage.

23. McMeekin, D. P. et al. A mixed-cation lead mixedhalide perovskite absorber for tandem solar cells. Science 351, 151-155 (2016).

24. Beal, R. E. et al. Cesium lead halide perovskites with improved stability for tandem solar cells. J. Phys. Chem. Lett. 7, 746-751 (2016).

25. Brivio, F., Caetano, C. \& Walsh, A. Thermodynamic origin of photoinstability in the $\mathrm{CH}_{3} \mathrm{NH}_{3} \mathrm{~Pb}\left(\mathrm{I}_{1-x} \mathrm{Br}_{x}\right)_{3}$ hybrid halide perovskite alloy. J. Phys. Chem. Lett. 7 1083-1087 (2016)

26. Bischak, C. G. et al. Origin of reversible photoinduced phase separation in hybrid perovskites. Nano Lett. 17 1028-1033 (2017).

27. Draguta, S. et al. Rationalizing the light-induced phase separation of mixed halide organic-inorganic perovskites. Nat Commun 8, 200 (2017).

28. Wang, X. et al. Suppressed phase separation of mixed halide perovskites confined in endotaxial matrices. Nat. Commun. 10, 695 (2019)

29. Scheidt, R. A. \& Kamat, P. V. Temperature-driven anion migration in gradient halide perovskites. J. Chem. Phys. 151, 134703 (2019).

30. Elmelund, T., Scheidt, R. A., Seger, B. \& Kamat, P. V. Bidirectional halide ion exchange in paired lead halide perovskite films with thermal activation. ACS Energy Lett. 4, 1961-1969 (2019).

31. Vicente, J. R. \& Chen, J. Phase segregation and photothermal remixing of mixed-halide lead perovskites. J. Phys. Chem. Lett. 11, 1802-1807 (2020).

32. Elmelund, T., Seger, B., Kuno, M. \& Kamat, P. V. how interplay between photo and thermal activation dictates halide ion segregation in mixed halide perovskites. ACS Energy Lett. 5, 56-63 (2020).

33. Bischak, C. G. et al. Tunable polaron distortions control the extent of halide demixing in lead halide perovskites. J. Phys. Chem. Lett. 9, 3998-4005 (2018).

34. Mao, W. et al. Light-induced reversal of ion segregation in mixed-halide perovskites. Nat. Mater. 20, 55-61 (2020).

35. Wright, A. D. et al. Electron-phonon coupling in hybrid lead halide perovskites. Nat. Commun. 7 , 11755 (2016).

36. Azpiroz, J. M., Mosconi, E., Bisquert, J. \& De Angelis, F Defect migration in methylammonium lead iodide and its role in perovskite solar cell operation. Energy Environ. Sci. 8, 2118-2127 (2015).

37. Meloni, S. et al. Ionic polarization-induced currentvoltage hysteresis in $\mathrm{CH}_{3} \mathrm{NH}_{3} \mathrm{PbX}_{3}$ perovskite solar cells. Nat. Commun. 7, 10334 (2016)

38. Haruyama, J., Sodeyama, K., Han, L. \& Tateyama, Y. First-principles study of ion diffusion in perovskite solar cell sensitizers. J. Am. Chem. Soc. 137 10048-10051 (2015).

39. Eames, C. et al. Ionic transport in hybrid lead iodide perovskite solar cells. Nat. Commun. 6, 7497 (2015)

40. Oranskaia, A., Yin, J., Bakr, O. M., Brédas, J. L. \& Mohammed, O. F. Halogen migration in hybrid perovskites: the organic cation matters. J. Phys. Chem. Lett. 9, 5474-5480 (2018).

41. Barboni, D. \& De Souza, R. A. The thermodynamics and kinetics of iodine vacancies in the hybrid perovskite methylammonium lead iodide. Energy Environ. Sci. 11, 3266-3274 (2018). This work shows that the interaction between photocarriers and iodide ions increases iodine vacancies in perovskites and thus the ionic conductivity

42. Motti, S. G. et al. Defect activity in lead halide perovskites. Adv. Mater. 31, 1901183 (2019). This work focuses on understanding the nature and photochemistry of defects in perovskite materials through a combination of density functional theory calculations and photophysics characterization

43. Samu, G. F. et al. Electrochemical hole injection selectively expels iodide from mixed halide perovskite films. J. Am. Chem. Soc. 141, 10812-10820 (2019).

44. DuBose, J. T. \& Kamat, P. V. $\mathrm{TiO}_{2}$-assisted halide ion segregation in mixed halide perovskite films. J. Am. Chem. Soc. 142, 5362-5370 (2020).

45. Belisle, R. A. et al. Impact of surfaces on photoinduced halide segregation in mixed-halide perovskites. ACS Energy Lett. 3, 2694-2700 (2018)

46. Lin, Y. et al. Excess charge-carrier induced instability of hybrid perovskites. Nat. Commun. 9, 4981 (2018)

47. Meggiolaro, D. et al. lodine chemistry determines the defect tolerance of lead-halide perovskites. Energy Environ. Sci. 11, 702-713 (2018).

48. Motti, S. G. et al. Controlling competing photochemical reactions stabilizes perovskite solar cells. Nat. Photonics 13, 532-539 (2019).

This work shows that photoinstabilities are related to the light-induced formation and annihilation of defects acting as carrier trap states.

49. Barker, A. J. et al. Defect-assisted photoinduced halide segregation in mixed-halide perovskite thin films. ACS Energy Lett. 2, 1416-1424 (2017).

50. Mosconi, E. \& De Angelis, F. Mobile ions in organohalide perovskites: interplay of electronic structure and dynamics. ACS Energy Lett. 1, 182-188 (2016).

51. Mao, W. et al. Visualizing phase segregation in mixedhalide perovskite single crystals. Angew. Chem. 58, 2893-2898 (2018).

52. Chen, W., Mao, W., Bach, U., Jia, B. \& Wen, X. Tracking dynamic phase segregation in mixed-halide perovskite single crystals under two-photon scanning laser illumination. Small Methods 3, 1900273 (2019).

53. Tang, X. et al. Local observation of phase segregation in mixed-halide perovskite. Nano Lett. 18 2172-2178 (2018)

54. Ohmann, R. et al. Real-space imaging of the atomic structure of organic-inorganic perovskite. J. Am. Chem. Soc. 137, 16049-16054 (2015).

55. She, L., Liu, M. \& Zhong, D. Atomic structures of $\mathrm{CH}_{3} \mathrm{NH}_{3} \mathrm{Pbl}_{3}(001)$ surfaces. ACS Nano 10 , $1126-1131$ (2016)

56. Ono, L. K. \& Qi, Y. Surface and interface aspects of organometal halide perovskite materials and solar cells. J. Phys. Chem. Lett. 7, 4764-4794 (2016).

57. Uratani, H. \& Yamashita, K. Charge carrier trapping at surface defects of perovskite solar cell absorbers: a first-principles study. J. Phys. Chem. Lett. 8, 742-746 (2017).

58. Meggiolaro, D., Mosconi, E. \& De Angelis, F. Formation of surface defects dominates ion migration in lead-halide perovskites. ACS Energy Lett. 4, 779-785 (2019)

This work finds that the formation and migration energy barrier of halide defects on perovskite surfaces can vary substantially depending on the surface termination.

59. Cai, L., She, L., Qin, H., Xu, L. \& Zhong, D. Monolayer methylammonium lead iodide films deposited on Au(111). Surf. Sci. 675, 78-82 (2018).

60. Castro-Méndez, A., Hidalgo, J. \& Correa-Baena, J. The role of grain boundaries in perovskite solar cells. Adv. Energy Mater. 0, 1901489 (2019).

61. Shao, Y. et al. Grain boundary dominated ion migration in polycrystalline organic-inorganic halide perovskite films. Energy Environ. Sci. 9, 1752-1759 (2016).

62. DeQuilettes, D. W. et al. Photo-induced halide redistribution in organic-inorganic perovskite films. Nat. Commun. 7, 11683 (2016). 
63. Knight, A. J. et al. Electronic traps and phase segregation in lead mixed-halide perovskite. ACS Energy Lett. 4, 75-84 (2018).

64. Knight, A. J., Patel, J. B., Snaith, H. J., Johnston, M. B. \& Herz, L. M. Trap states, electric fields, and phase segregation in mixed-halide perovskite photovoltaic devices. Adv. Energy Mater. 10, 1903488 (2020). This work focuses on understanding trap behaviour under illumination and electrical fields, elucidating the possible role of traps in halide segregation.

65. Kim M., Motti S. G., Sorrentino R. \& Petrozza A. Enhanced solar cell stability by hygroscopic polymer passivation of metal halide perovskite thin film. Energ. Environ. Sci.11, 2609-2619 (2018)

66. Yin, W., Shi, T. \& Yan, Y. Unusual defect physics in $\mathrm{CH}_{3} \mathrm{NH}_{3} \mathrm{Pbl}_{3}$ perovskite solar cell absorber. Appl. Phys. Lett. 104, 63903 (2014).

67. Srimath Kandada, A. R. et al. Nonlinear carrier interactions in lead halide perovskites and the role of defects. J. Am. Chem. Soc. 138, 13604-13611 (2016).

68. Motti, S. G. et al. Photoinduced emissive trap states in lead halide perovskite semiconductors. ACS Energy Lett. 1, 726-730 (2016)

69. Li, W. et al. Control of charge recombination in perovskites by oxidation state of halide vacancy. J. Am. Chem. Soc. 140, 15753-15763 (2018)

70. Leijtens, T. et al. Carrier trapping and recombination: the role of defect physics in enhancing the open circuit voltage of metal halide perovskite solar cells. Energy Environ. Sci. 9, 3472-3481 (2016).

71. $\mathrm{Ni}, \mathrm{Z}$. et al. Resolving spatial and energetic distributions of trap states in metal halide perovskite solar cells. Science 367, 1352 (2020). By studying the drive-level capacitance profiling of perovskites, this work shows that most of the deep traps are located at the crystal surface.

72. Park, B. et al. Understanding how excess lead iodide precursor improves halide perovskite solar cell performance. Nat. Commun. 9, 3301 (2018).

73. Zhao, Y. et al. Double-side-passivated perovskite solar cells with ultra-low potential loss. Sol. RRL 3 1800296 (2018)

74. Jacobsson, T. J. et al. Unreacted $\mathrm{Pbl}_{2}$ as a doubleedged sword for enhancing the performance of perovskite solar cells. J. Am. Chem. Soc. 138 10331-10343 (2016)

75. Cho, H. et al. Overcoming the electroluminescence efficiency limitations of perovskite light-emitting diodes. Science 350, 1222 (2015).

76. Cao, Y. et al. Perovskite light-emitting diodes based on spontaneously formed submicrometre-scale structures. Nature 562, 249-253 (2018).

77. Lin, K. et al. Perovskite light-emitting diodes with external quantum efficiency exceeding 20 percent. Nature 562, 245-248 (2018)

78. Yuan, Z. et al. Unveiling the synergistic effect of precursor stoichiometry and interfacial reactions for perovskite light-emitting diodes. Nat. Commun. 10, 2818 (2019)

79. Jia, Y. et al. Role of excess FAl in formation of high-efficiency $\mathrm{FAPbl}_{3}$-based light-emitting diodes. Adv. Funct. Mater. 30, 1906875 (2020)

80. Merdasa, A. et al. Impact of excess lead iodide on the recombination kinetics in metal halide perovskites. ACS Energy Lett. 4, 1370-1378 (2019).

81. Fu, F. et al. $\mathrm{I}_{2}$ vapor-induced degradation of formamidinium lead iodide based perovskite solar cells under heat-light soaking conditions. Energy Environ. Sci. 12, 3074-3088 (2019).

82. Long, M. et al. Abnormal synergetic effect of organic and halide ions on the stability and optoelectronic properties of a mixed perovskite via in situ characterizations. Adv. Mater. 30, 1801562 (2018).

83. Gratia, P. et al. intrinsic halide segregation at nanometer scale determines the high efficiency of mixed cation/mixed halide perovskite solar cells J. Am. Chem. Soc. 138, 15821-15824 (2016).

84. Correa-Baena, J. et al. Homogenized halides and alkali cation segregation in alloyed organic-inorganic perovskites. Science 363, 627 (2019).

85. Mahesh, S. et al. Revealing the origin of voltage loss in mixed-halide perovskite solar cells. Energy Environ. Sci. 13, 258-267 (2020). This work reveals that imperfections within the mixed I-Br perovskite, rather than halide segregation, dominate the open-circuit voltage loss in wide-bandgap perovskite solar cells.

86. Peña-Camargo, F. et al. Halide segregation versus interfacial recombination in bromide-rich wide-gap perovskite solar cells. ACS Energy Lett. $\mathbf{5}$ 2728-2736 (2020).
87. Tao, S. et al. Absolute energy level positions in tin- and lead-based halide perovskites. Nat. Commun. 10 2560 (2019).

88. Lin, Y. et al. Matching charge extraction contact for wide-bandgap perovskite solar cells. Adv. Mater. 29 1700607 (2017)

89. Hutter, E. M. et al. Thermodynamic stabilization of mixed-halide perovskites against phase segregation Cell Rep. Phys. Sci. 1, 100120 (2020).

90. Tan, H. et al. Dipolar cations confer defect tolerance in wide-bandgap metal halide perovskites. Nat. Commun 9, 3100 (2018)

91. Frolova, L. A. et al. Highly efficient all-inorganic planar heterojunction perovskite solar cells produced by thermal coevaporation of Csl and $\mathrm{Pbl}_{2}$. J. Phys. Chem. Lett. 8, 67-72 (2017)

92. Dastidar, S. et al. Quantitative phase-change thermodynamics and metastability of perovskitephase cesium lead iodide. J. Phys. Chem. Lett. 8, 1278-1282 (2017).

93. Chen, C. et al. All-vacuum-deposited stoichiometrically balanced inorganic cesium lead halide perovskite solar cells with stabilized efficiency exceeding $11 \%$. Adv. Mater. 29, 1605290 (2017).

94. Tai, Q., Tang, K. \& Yan, F. Recent progress of inorganic perovskite solar cells. Energy Environ. Sci. 12, 2375-2405 (2019).

95. Ho-Baillie, A., Zhang, M., Lau, C. F. J., Ma, F. \& Huang, S. Untapped potentials of inorganic metal halide perovskite solar cells. Joule 3, 938-955 (2019).

96. Faheem, M. B. et al. All-inorganic perovskite solar cells: energetics, key challenges, and strategies toward commercialization. ACS Energy Lett. 5, 290-320 (2020).

97. Hörantner, M. T. et al. The potential of multi-junction perovskite solar cells. ACS Energy Lett. 2, 2506-2513 (2017).

98. Aydin, E. et al. Interplay between temperature and bandgap energies on the outdoor performance of perovskite/silicon tandem solar cells. Nat. Energy 5, 851-859 (2020).

99. Hu, M., Bi, C., Yuan, Y., Bai, Y. \& Huang, J. Stabilized wide bandgap $\mathrm{MAPbBr}_{x} \mathrm{I}_{3-x}$ perovskite by enhanced grain size and improved crystallinity. Adv. Sci. 3 , 1500301 (2016)

100. Yu, Y. et al. Synergistic effects of lead thiocyanate additive and solvent annealing on the performance of wide-bandgap perovskite solar cells. ACS Energy Lett. 2, 1177-1182 (2017).

101. Zhou, Y. et al. Composition-tuned wide bandgap perovskites: from grain engineering to stability and performance improvement. Adv. Funct. Mater. 28 1803130 (2018)

102. Son, D. et al. Self-formed grain boundary healing layer for highly efficient $\mathrm{CH}_{3} \mathrm{NH}_{3} \mathrm{Pbl}_{3}$ perovskite solar cells. Nat. Energy 1, 16081 (2016).

103. Chen, S. et al. Spatial distribution of lead iodide and local passivation on organo-lead halide perovskite. ACS Appl. Mater. Inter. 9, 6072-6078 (2017).

104. Jiang, Q. et al. Planar-structure perovskite solar cells with efficiency beyond 21\%. Adv. Mater. 29, 1703852 (2017).

105. Li, Q. et al. Efficient perovskite solar cells fabricated through $\mathrm{CsCl}$-enhanced $\mathrm{Pbl}_{2}$ precursor via sequential deposition. Adv. Mater. 30, 1803095 (2018).

106. Abdi-Jalebi, M. et al. Maximizing and stabilizing luminescence from halide perovskites with potassium passivation. Nature 555, 497 (2018)

107. Wang, Z. et al. Efficient ambient-air-stable solar cells with 2D-3D heterostructured butylammoniumcaesium-formamidinium lead halide perovskites. Nat. Energy 6, 135 (2017).

108. Gharibzadeh, S. et al. Record open-circuit voltage wide-bandgap perovskite solar cells utilizing 2D/3D perovskite heterostructure. Adv. Energy Mater. 9 , 1803699 (2019)

109. Wang, F. et al. Phenylalkylamine passivation of organolead halide perovskites enabling high-efficiency and air-stable photovoltaic cells. Adv. Mater. 28, 9986-9992 (2016).

110. Zhou, Y. et al. Benzylamine-treated wide-bandgap perovskite with high thermal-photostability and photovoltaic performance. Adv. Energy Mater. 7, 1701048 (2017)

111. Lei, Y. et al. A fabrication process for flexible singlecrystal perovskite devices. Nature $\mathbf{5 8 3}, \mathbf{7 9 0 - 7 9 5}$ (2020).

112. Chen, Z. et al. Single-crystal $\mathrm{MAPbl}_{3}$ perovskite solar cells exceeding $21 \%$ power conversion efficiency. ACS Energy Lett. 4, 1258-1259 (2019).
113. Chung, I. et al. CsSnl ${ }_{3}$ : semiconductor or metal? High electrical conductivity and strong near-infrared photoluminescence from a single material. High hole mobility and phase-transitions. J. Am. Chem. Soc. 134, 8579-8587 (2012).

114. Wang, F. et al. Organic cation-dependent degradation mechanism of organotin halide perovskites. Adv. Funct. Mater. 26, 3417-3423 (2016).

115. Shi, T. et al. Effects of organic cations on the defect physics of tin halide perovskites. J. Mater. Chem. A 5, 15124-15129 (2017).

116. Meggiolaro, D., Ricciarelli, D., Alasmari, A A. Alasmary, F. A. S. \& De Angelis, F. Tin versus lead redox chemistry modulates charge trapping and self-doping in tin/lead iodide perovskites. J. Phys. Chem. Lett. 11, 3546-3556 (2020).

This work reveals that lead iodide perovskites are dominated by iodine chemistry, whereas tin chemistry dominates the defect chemistry of tin iodide perovskites.

117. Xu, P., Chen, S., Xiang, H., Gong, X. \& Wei, S. Influence of defects and synthesis conditions on the photovoltaic performance of perovskite semiconductor $\mathrm{CsSnl}_{3}$. Chem. Mater. 26, 6068-6072 (2014).

118. Nasti, G. \& Abate, A. Tin halide perovskite $\left(\mathrm{ASnX}_{3}\right)$ solar cells: a comprehensive guide toward the highest power conversion efficiency. Adv. Energy Mater. 10, 1902467 (2020).

119. Parrott, E. S. et al. Effect of structural phase transition on charge-carrier lifetimes and defects in $\mathrm{CH}_{3} \mathrm{NH}_{3} \mathrm{Snl}$ perovskite. J. Phys. Chem. Lett. 7, 1321-1326 (2016).

120. Noel, N. K. et al. Lead-free organic-inorganic tin halide perovskites for photovoltaic applications Energy Environ. Sci. 7, 3061-3068 (2014).

121. Kumar, M. H. et al. Lead-free halide perovskite solar cells with high photocurrents realized through vacancy modulation. Adv. Mater. 26, 7122-7127 (2014).

122. Milot, R. L. et al. The effects of doping density and temperature on the optoelectronic properties of formamidinium tin triiodide thin films. Adv. Mater. 30 1804506 (2018)

123. Wu, B. et al. Long minority-carrier diffusion length and low surface-recombination velocity in inorganic lead-free $\mathrm{CsSnl}_{3}$ perovskite crystal for solar cells. Adv. Funct. Mater. 27, 1604818 (2017).

124. Ma, L. et al. Carrier diffusion lengths of over $500 \mathrm{~nm}$ in lead-free perovskite $\mathrm{CH}_{3} \mathrm{NH}_{3} \mathrm{Snl}_{3}$ films. J. Am. Chem. Soc. 138, 14750-14755 (2016).

125. Nishimura, K. et al. Lead-free tin-halide perovskite solar cells with $13 \%$ efficiency. Nano Energy $\mathbf{7 4}$, 104858 (2020).

126. Milot, R. L., Eperon, G. E., Snaith, H. J., Johnston, M. B. \& Herz, L. M. Temperature-dependent charge-carrier dynamics in $\mathrm{CH}_{3} \mathrm{NH}_{3} \mathrm{Pbl}_{3}$ perovskite thin films. Adv. Funct. Mater. 25, 6218-6227 (2015).

127. Marshall, K. P. Walton, R. I. \& Hatton, R. A. Tin perovskite/fullerene planar layer photovoltaics: improving the efficiency and stability of lead-free devices. J. Mater. Chem. A 3, 11631-11640 (2015).

128. Tsai, C. et al. Role of tin chloride in tin-rich mixedhalide perovskites applied as mesoscopic solar cells with a carbon counter electrode. ACS Energy Lett. 1 , 1086-1093 (2016).

129. Tai, Q. et al. Antioxidant grain passivation for airstable tin-based perovskite solar cells. Angew. Chem. 58, 806-810 (2019)

130. Gupta, S., Cahen, D. \& Hodes, G. How SnF $\mathrm{F}_{2}$ impacts the material properties of lead-free tin perovskites. J. Phys. Chem. C. 122, 13926-13936 (2018).

131. Lee, S. J. et al. Fabrication of efficient formamidinium tin iodide perovskite solar cells through $\mathrm{SnF}_{2}$-pyrazine complex. J. Am. Chem. Soc. 138, 3974-3977 (2016).

132. Song, T. et al. Piperazine suppresses self-doping in $\mathrm{CsSnl}_{3}$ perovskite solar cells. ACS Appl. Energy Mater 1, 4221-4226 (2018).

133. Song, T. et al. Importance of reducing vapor atmosphere in the fabrication of tin-based perovskite solar cells. J. Am. Chem. Soc. 139, 836-842 (2017).

134. Gu, F. et al. Improving performance of lead-free formamidinium tin triiodide perovskite solar cells by tin source purification. Sol. RRL 2, 1800136 (2018).

135. Zhao, Z. et al. Mixed-organic-cation tin iodide for lead-free perovskite solar cells with an efficiency of $8.12 \%$. Adv. Sci. 4, 1700204 (2017).

136. Li, F. et al. A cation-exchange approach for the fabrication of efficient methylammonium tin iodide perovskite solar cells. Angew. Chem. Int. Ed. 58, 6688-6692 (2019). 
137. Jokar, E., Chien, C., Tsai, C., Fathi, A. \& Diau, E. W. Robust tin-based perovskite solar cells with hybrid organic cations to attain efficiency approaching 10\%. Adv. Mater. 31, 1804835 (2019).

138. Spanopoulos, I. et al. Unraveling the chemical nature of the 3D 'hollow' hybrid halide perovskites. J. Am. Chem. Soc. 140, 5728-5742 (2018).

139. Ke, W., Stoumpos, C. C. \& Kanatzidis, M. G. 'Unleaded' perovskites: status quo and future prospects of tin-based perovskite solar cells. Adv. Mater. 31 1803230 (2019).

140. Takahashi, Y. Hasegawa, H., Takahashi, Y. \& Inabe, T. Hall mobility in tin iodide perovskite $\mathrm{CH}_{3} \mathrm{NH}_{3} \mathrm{Snl}_{3}$ : evidence for a doped semiconductor. J. Solid State Chem. 205, 39-43 (2013).

141. Gu, S. et al. Tin and mixed lead-tin halide perovskite solar cells: progress and their application in tandem solar cells. Adv. Mater. 32, 1907392 (2020).

142. Diau, E. W., Jokar, E. \& Rameez, M. Strategies to improve performance and stability for tin-based perovskite solar cells. ACS Energy Lett. 4 1930-1937 (2019).

143. Liao, M. et al. Efficient and stable FASnl ${ }_{3}$ perovskite solar cells with effective interface modulation by low-dimensional perovskite layer. ChemSusChem 12 , 5007-5014 (2019).

144. Chen, K. et al. Low-dimensional perovskite interlayer for highly efficient lead-free formamidinium tin iodide perovskite solar cells. Nano Energy 49, 411-418 (2018).
145. Liu, X., Wang, Y., Xie, F., Yang, X. \& Han, L. Improving the performance of inverted formamidinium tin iodide perovskite solar cells by reducing the energy-level mismatch. ACS Energy Lett. 3, 1116-1121 (2018).

146. Xiao, Q. et al. Dopant-free squaraine-based polymeric hole-transporting materials with comprehensive passivation effects for efficient all-inorganic perovskite solar cells. Angew. Chem. 58, 17724-17730 (2019).

147. $\mathrm{Ke}, \mathrm{W}$. et al. $\mathrm{TiO}_{2}-\mathrm{ZnS}$ cascade electron transport layer for efficient formamidinium tin iodide perovskite solar cells. J. Am. Chem. Soc. 138, 14998-15003 (2016).

148. Hao, F., Stoumpos, C. C., Chang, R. P. H. \& Kanatzidis, M. G. Anomalous band gap behavior in mixed $\mathrm{Sn}$ and $\mathrm{Pb}$ perovskites enables broadening of absorption spectrum in solar cells. J. Am. Chem. Soc. 136, 8094-8099 (2014).

149. Leijtens, T., Prasanna, R., Gold-Parker, A., Toney, M. F. \& McGehee, M. D. Mechanism of tin oxidation and stabilization by lead substitution in tin halide perovskites. ACS Energy Lett. 2 , 2159-2165 (2017).

150. Prasanna, R. et al. Design of low bandgap tin-lead halide perovskite solar cells to achieve thermal, atmospheric and operational stability. Nat. Energy 4, 939-947 (2019).

151. Werner, J. et al. Improving low-bandgap tin-lead perovskite solar cells via contact engineering and gas quench processing. ACS Energy Lett. 5, 1215-1223 (2020).

\section{Acknowledgements}

This work has been funded by the European Union project PERT PV under grant agreement no. 763977 and ERC project SOPHY under grant agreement N 771528 .

\section{Author contributions}

Y.Z., I.P. and A.P. conceived the review structure. Y.Z. developed the section on high-bandgap semiconductors. I.P. developed the section on narrow-bandgap semiconductors. D.M. and F.D.A. reviewed the contribution to the field from theoretical studies. All authors contributed to the writing and revision of the manuscript.

\section{Competing interests}

The authors declare no competing interests.

\section{Peer review information}

Nature Reviews Materials thanks Jinsong Huang, Michael D. McGehee and the other, anonymous, reviewer(s) for their contribution to the peer review of this work.

\section{Publisher's note}

Springer Nature remains neutral with regard to jurisdictional claims in published maps and institutional affiliations.

\section{RELATED LINKS}

Currently certified 25.5\%: https://www.nrel.gov/pv/

cell-efficiency.html

(c) Springer Nature Limited 2021 